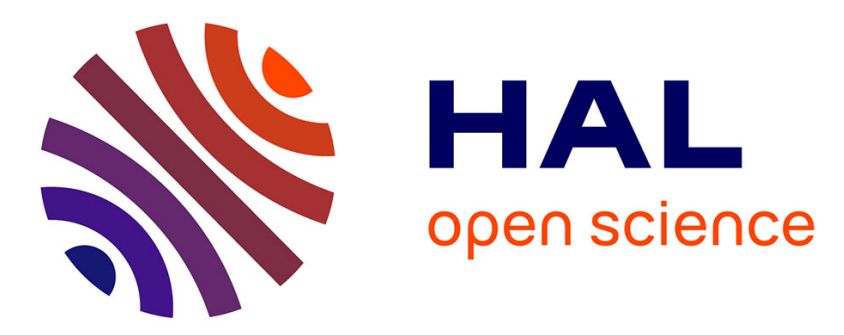

\title{
On the equivalence of financial structures with long-term assets
}

Jean-Marc Bonnisseau, Achis Chery

\section{- To cite this version:}

Jean-Marc Bonnisseau, Achis Chery. On the equivalence of financial structures with long-term assets. 2014. hal-01130785

\section{HAL Id: hal-01130785 https://hal.science/hal-01130785}

Submitted on 12 Mar 2015

HAL is a multi-disciplinary open access archive for the deposit and dissemination of scientific research documents, whether they are published or not. The documents may come from teaching and research institutions in France or abroad, or from public or private research centers.
L'archive ouverte pluridisciplinaire HAL, est destinée au dépôt et à la diffusion de documents scientifiques de niveau recherche, publiés ou non, émanant des établissements d'enseignement et de recherche français ou étrangers, des laboratoires publics ou privés. 


\section{Documents de Travail du Centre d'Economie de la Sorbonne}

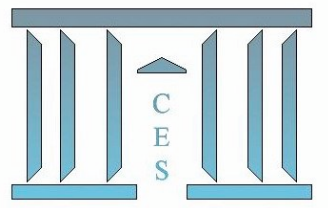

\section{On the equivalence of financial structures with long-term assets}

Jean-Marc BonnisseAU, Achis CHERY

2014.81

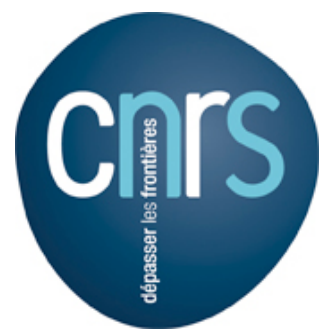




\title{
On the equivalence of financial structures with long-term assets.
}

\author{
Jean-Marc Bonnisseau*and Achis Chery ${ }^{\dagger}$
}

November 20, 2014

\begin{abstract}
In a stochastic financial exchange economy, two financial structures are equivalent if, for each given state price, regardless the associated arbitrage free price, the marketable payoffs are identical. The key property of two equivalent financial structures is that, when associated with any standard exchange economy, they lead to the same financial equilibrium. We exhibit a sufficient condition for the equivalence of two financial structures with possibly long-term assets. We then apply this result to financial structures built upon primitive assets and their re-trading to get a necessary and sufficient condition. We also borrow an assumption from [7] to prove the equivalence between a financial structure and its reduced forms and between to complete financial structures.
\end{abstract}

Keywords: Equivalent financial structures, financial equilibrium, multi-period model, long-term assets, financial sub-structure, reduced forms

*Paris School of Economics, Université Paris 1 Panthéon Sorbonne, 106-112 boulevard de l'Hôpital, 75647 Paris Cedex 13, France, Jean-marc.Bonnisseau@univ-paris1.fr

†Paris School of Economics, Université Paris 1 Panthéon Sorbonne, 106-112 boulevard de l'Hôpital, 75647 Paris Cedex 13, France et Centre de Recherche en Gestion et Economie du Développement (CREGED), Université Quisqueya, 218 Haut Turgeau, 6113 Port-au-prince, Haïti, Achis.Chery@malix.univ-paris1.fr 


\section{Contents}

1 Introduction $\quad 2$

2 Financial exchange economy and equilibrium 4

2.1 Time and uncertainty ................ 4

2.2 The financial structure . . . . . . . . . . . . . . 5

2.3 The stochastic exchange economy . . . . . . . . . . . 7

2.4 Financial equilibrium . . . . . . . . . . . . . . . 7

3 Equivalent financial structures $\quad 8$

3.1 Examples of equivalent financial stuctures . . . . . . . . . . . 9

4 Sufficient conditions for equivalence $\quad 10$

4.1 Equivalence under Assumption R1 . . . . . . . . . . . . . . . 12

4.2 Comments on Assumption R1 . . . . . . . . . . . . . . . 15

4.3 Equivalence with re-trading . . . . . . . . . . . . . . 16

4.4 Equivalence with reduced forms . . . . . . . . . . . . . . . 19

4.5 Equivalence for complete financial structures . . . . . . . . . . . 22

\section{Introduction}

We consider stochastic financial exchange economies defined on a given finite date-event tree representing time and uncertainty. The financial structures may include long-term assets. We study the equivalence relation on financial structures introduced in $[4,8]$, when the portfolios of agents are unconstrained. Two financial structures are equivalent if, for each state price, the marketable payoffs are the same for the arbitrage free asset prices associated to the given state price. In other words, this means that the ranges of the full payoff matrices are identical.

A financial structure allows economic agents to transfer wealth across nodes of the date-event tree through the marketable payoff set. Thereby given a spot price $p$, the budget set of an agent is fully determined by the marketable payoff set. So the budget sets are the same for two equivalent financial structures. The main consequence is that, regardless of the standard exchange economy $\Sigma$, the existence of a financial equilibrium in $\Sigma$ associated with a financial structure $\mathcal{F}$ is equivalent to the existence of equilibrium in $\Sigma$ associated with any other financial structure $\mathcal{F}^{\prime}$ belonging to the equivalence class of $\mathcal{F}$. Furthermore, the equilibrium consumption and the equilibrium spot price are the same, only the asset portfolios have to be suitably modified. Hence the importance of studying the notion of equivalence between the financial structures since the existence of a financial equilibrium for a given financial structure is extended to the equivalence class of this financial structure. 
Equivalent financial structures have been studied, among others, by AouaniCornet and Cornet-Ranjan [5, 8] in the two-period case. In [8], it is proved that two financial structures are equivalent when the ranges of their payoff matrices are equal. We have generalized this result to the multi-period case if all assets are short-term in [6]. By means of examples in Section 4, we show that, with long-term assets, equality between the images of payoff matrices of two financial structures is neither necessary nor sufficient to get the equivalence of these structures.

So, to get the equivalence with long-term assets, we introduce an additional assumptions, Assumptions R1, on the payoff matrices of two financial structures. Precisely, Assumption R1 means that, at each emission node $\xi$, the assets issued at node $\xi$ for the two structures offer the same possibilities of transfer for the successors of $\xi$. In other words, the marketable payoffs generated by the assets issued at the same node are identical for the two structures. For a two-period economy, this just means that the ranges of the payoff matrices are the same since there is a unique emission node. The main result of the chapter is that Assumption $\mathbf{R} \mathbf{1}$ is sufficient to get the equivalence of the financial structures. Nevertheless, note that Assumption R1 is not necessary to get the equivalence as illustrated in an example in Section 4.

We apply this result to the special case of a financial structures with retrading where assets are issued at date 0 and then re-traded at every node like in Magill-Quinzii [9]. In this case, note that a financial structure is fully described by the primitive payoff matrix of assets issued at date 0 . To do the link with the previous model, following [1], we introduce the re-trading extension of the financial structure by considering that the re-trade of an asset is equivalent to issuing a new asset. We prove that the equality between the ranges of the two primitive payoff matrices of two financial structures with re-trading is equivalent with the fact that they satisfy Assumption R1, so implies the equivalence of their re-trading extensions.

Then, we study the equivalence of a financial structure with their reduced forms. A reduced form is obtained by removing the redundant assets. This concept is extensively studied in the two period case in $[3,4,2]$. The interest for studying this question comes from the methodology to prove the existence of a financial equilibrium. Indeed, we need a fixed point argument, so we need the compactness of attainable portfolios. With a financial structure, we may not have bounded attainable portfolios due to the presence of redundant assets. So, a way to get an equilibrium is: considering a reduced form by removing redundant assets; obtaining bounded attainable portfolios for the reduced form; proving the existence of an equilibrium for the reduced form; getting an equilibrium for the original economy by equivalence.

We provide an example showing that a structure may not be equivalent with their reduced form. To get the equivalence, we borrow Assumption $\mathbf{R}$ from [7]. A financial structure satisfies Asssumption $\mathbf{R}$ if the returns of the assets issued at a node $\xi$ are not redundant with the returns of the assets issued previously. We show that a financial structure satisfying this assumption is equivalent to their reduced forms.

Finally, using a result of [7], we prove that two complete financial structures 
are equivalent if both satisfy Assumption R.

In Section 2, we describe the general framework of a financial exchange economy and we define a financial equilibrium. In Section 3, we state the definition of equivalence between two financial structures and we prove the key result on the link between financial equilibrium for two equivalent financial structures. In Section 4, we present and comment our key assumption R1, we prove the equivalence under $\mathbf{R} \mathbf{1}$ and then we develop the applications for the re-trading case, the reduced forms and finally the complete financial structures.

\section{Financial exchange economy and equilibrium}

In this section, we present the model and the notations, which are borrowed from Angeloni-Cornet[1] and are essentially the same as those of Magill-Quinzii[9].

\subsection{Time and uncertainty}

$W^{1}$ consider a multi-period exchange economy with $(T+1)$ dates, $t \in \mathcal{T}:=$ $\{0, \ldots, T\}$, and a finite set of agents $\mathcal{I}$. The uncertainty is described by a dateevent tree $\mathbb{D}$ of length $T+1$. The set $\mathbb{D}_{t}$ is the set of nodes (also called dateevents) that could occur at date $t$ and the family $\left(\mathbb{D}_{t}\right)_{t \in \mathcal{T}}$ defines a partition of the set $\mathbb{D}$; for each $\xi \in \mathbb{D}$, we denote by $t(\xi)$ the unique date $t \in \mathcal{T}$ such that $\xi \in \mathbb{D}_{t}$.

At date $t=0$, there is a unique node $\xi_{0}$, that is $\mathbb{D}_{0}=\left\{\xi_{0}\right\}$. As $\mathbb{D}$ is a tree, each node $\xi$ in $\mathbb{D} \backslash\left\{\xi_{0}\right\}$ has a unique immediate predecessor denoted $\operatorname{pr}(\xi)$ or $\xi^{-}$. The mapping $p r$ maps $\mathbb{D}_{t}$ to $\mathbb{D}_{t-1}$. Each node $\xi \in \mathbb{D} \backslash \mathbb{D}_{T}$ has a set of immediate successors defined by $\xi^{+}=\left\{\bar{\xi} \in \mathbb{D}: \xi=\bar{\xi}^{-}\right\}$.

For $\tau \in \mathcal{T} \backslash\{0\}$ and $\xi \in \mathbb{D} \backslash \cup_{t=0}^{\tau-1} \mathbb{D}_{t}$, we define $p r^{\tau}(\xi)$ by the recursive formula: $p r^{\tau}(\xi)=p r\left(p r^{\tau-1}(\xi)\right)$. We then define the set of successors and the set of predecessors of $\xi$ as follows:

$$
\begin{aligned}
& \mathbb{D}^{+}(\xi)=\left\{\xi^{\prime} \in \mathbb{D}: \exists \tau \in \mathcal{T} \backslash\{0\} \mid \xi=p r^{\tau}\left(\xi^{\prime}\right)\right\} \\
& \mathbb{D}^{-}(\xi)=\left\{\xi^{\prime} \in \mathbb{D}: \exists \tau \in \mathcal{T} \backslash\{0\} \mid \xi^{\prime}=p r^{\tau}(\xi)\right\}
\end{aligned}
$$

For each $\xi \in \mathbb{D}$, we note by $\mathbb{D}(\xi)$ the union of $\xi$ with $\mathbb{D}^{+}(\xi)$. If $\xi^{\prime} \in \mathbb{D}^{+}(\xi)$ [resp. $\xi^{\prime} \in \mathbb{D}(\xi)$ ], we use the notation $\xi^{\prime}>\xi$ [resp. $\xi^{\prime} \geq \xi$ ]. Note that $\xi^{\prime} \in \mathbb{D}^{+}(\xi)$ if and only if $\xi \in \mathbb{D}^{-}\left(\xi^{\prime}\right)$ and similarly $\xi^{\prime} \in \xi^{+}$if and only if $\xi=\left(\xi^{\prime}\right)^{-}$.

\footnotetext{
${ }^{1}$ We use the following notations. A $(\mathbb{D} \times \mathcal{J})$-matrix $A$ is an element of $\mathbb{R}^{\mathbb{D} \times \mathcal{J}}$, with entries $\left(a_{\xi}^{j}\right)_{(\xi \in \mathbb{D}, j \in \mathcal{J})}$; we denote by $A_{\xi} \in \mathbb{R}^{\mathcal{J}}$ the $\xi$-th row of $A$ and by $A^{j} \in \mathbb{R}^{\mathbb{D}}$ the $j$-th column of $A$. We recall that the transpose of $A$ is the unique $(\mathcal{J} \times \mathbb{D})$-matrix ${ }^{t} \mathrm{~A}$ satisfying $(A x) \bullet \mathbb{D} y=$ $x \bullet \mathcal{J}\left({ }^{t} \mathrm{Ay}\right)$ for every $x \in \mathbb{R}^{\mathcal{J}}, y \in \mathbb{R}^{\mathbb{D}}$, where $\bullet \mathbb{D}\left[\right.$ resp. $\left.\bullet_{\mathcal{J}}\right]$ denotes the usual inner product in $\mathbb{R}^{\mathbb{D}}\left[\right.$ resp. $\left.\mathbb{R}^{\mathcal{J}}\right]$. We denote by $\operatorname{rank} A$ the rank of the matrix $A$ and by Vect $(A)$ the range of $A$, that is the linear sub-space spanned by the column vectors of $A$. For every subset $\tilde{\mathbb{D}} \subset \mathbb{D}$ and $\tilde{\mathcal{J}} \subset \mathcal{J}$, the matrix $A_{\tilde{\mathbb{D}}}^{\tilde{\mathcal{J}}}$ is the $(\tilde{\mathbb{D}} \times \tilde{\mathcal{J}})$-sub-matrix of $A$ with entries $a_{\xi}^{j}$ for every $(\xi, j) \in(\tilde{\mathbb{D}} \times \tilde{\mathcal{J}})$. Let $x, y$ be in $\mathbb{R}^{n} ; x \geq y$ (resp. $x \gg y$ ) means $x_{h} \geq y_{h}\left(\right.$ resp. $x_{h}>y_{h}$ ) for every $h=1, \ldots, n$ and we let $\mathbb{R}^{n}=\left\{x \in \mathbb{R}^{n}: x \geq 0\right\}, \mathbb{R}^{n}=\left\{x \in \mathbb{R}^{n}: x \gg 0\right\}$. We also use the notation $x>y$ if $x \geq y$ and $x \neq y$. The Euclidean norm in the different Euclidean spaces is denoted $\|\cdot\|$ and the closed ball centered at $x$ and of radius $r>0$ is denoted $\bar{B}(x, r):=\left\{y \in \mathbb{R}^{n} \mid\|y-x\| \leq r\right\}$.
} 


\section{A simple example of $\mathbb{D}$}

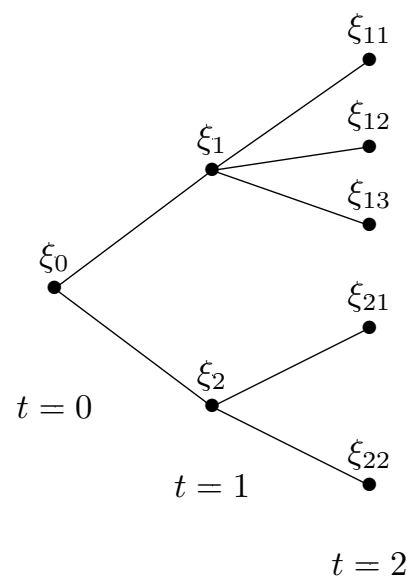

Figure 1: the tree $\mathbb{D}$

Here: $\mathbb{D}=\left\{\xi_{0}, \xi_{1}, \xi_{2}, \xi_{11}, \xi_{12}, \xi_{13}, \xi_{21}, \xi_{22}\right\}, T=2$, the length of $\mathbb{D}$ is 3 , $\mathbb{D}_{2}=\left\{\xi_{11}, \xi_{12}, \xi_{13}, \xi_{21}, \xi_{22}\right\}, \xi_{1}^{+}=\left\{\xi_{11}, \xi_{12}, \xi_{13}\right\}, \mathbb{D}^{+}\left(\xi_{2}\right)=\left\{\xi_{21}, \xi_{22}\right\}, t\left(\xi_{11}\right)=$ $t\left(\xi_{12}\right)=t\left(\xi_{13}\right)=t\left(\xi_{21}\right)=t\left(\xi_{22}\right)=2, \mathbb{D}^{-}\left(\xi_{11}\right)=\left\{\xi_{0}, \xi_{1}\right\}$.

\subsection{The financial structure}

At each node $\xi \in \mathbb{D}$, there is a spot market on which a finite set $\mathbb{H}=\{1, \ldots, H\}$ of divisible and physical goods are exchanged. We assume that each good is perishable, that is, its life does not last more than one date. In this model, a commodity is a pair $(h, \xi)$ of a physical good $h \in \mathbb{H}$ and the node $\xi \in \mathbb{D}$ at which the good is available. Then the commodity space is $\mathbb{R}^{\mathbb{L}}$, where $\mathbb{L}=\mathbb{H} \times \mathbb{D}$. An element $x \in \mathbb{R}^{\mathbb{L}}$ is called a consumption, that is to say $x=(x(\xi))_{\xi \in \mathbb{D}} \in \mathbb{R}^{\mathbb{L}}$, where $x(\xi)=(x(h, \xi))_{h \in \mathbb{H}} \in \mathbb{R}^{\mathbb{H}}$ for each $\xi \in \mathbb{D}$.

We denote by $p=(p(\xi))_{\xi \in \mathbb{D}} \in \mathbb{R}^{\mathbb{L}}$ the vector of spot prices and $p(\xi)=$ $(p(h, \xi))_{h \in \mathbb{H}} \in \mathbb{R}^{\mathbb{H}}$ is called the spot price at node $\xi$. The spot price $p(h, \xi)$ is the price at the node $\xi$ for immediate delivery of one unit of the physical good $h$. Thus the value of a consumption $x(\xi)$ at node $\xi \in \mathbb{D}$ (measured in unit account of the node $\xi$ ) is

$$
p(\xi) \bullet \mathbb{H} x(\xi)=\sum_{h \in \mathbb{H}} p(h, \xi) x(h, \xi) .
$$

The financial structure is constituted by a finite set of assets denoted $\mathcal{J}=$ $\{1, \ldots, J\}$. An asset $j \in \mathcal{J}$ is a contract issued at a given and unique node in $\mathbb{D}$ denoted $\xi(j)$ and called issuance node of $j$. Each asset is bought or sold only at its issuance node $\xi(j)$ and yields payoffs only at the successor nodes $\xi^{\prime}$ of $\mathbb{D}^{+}(\xi(j))$. To simplify the notation, we consider the payoff of asset $j$ at every node $\xi \in \mathbb{D}$ and we assume that it is zero if $\xi$ is not a successor of the issuance node $\xi(j)$. The payoff may depend upon the spot price vector $p \in \mathbb{R}^{\mathbb{L}}$ and is 
denoted by $V_{\xi}^{j}(p)$. Formally, we assume that $V_{\xi}^{j}(p)=0$ if $\xi \notin \mathbb{D}^{+}(\xi(j))$. An asset is a short term asset if it has a non-zero payoff only at the immediate successors of the issuance node, that is, $V_{\xi^{\prime}}^{j}(p)=0$ if $\xi^{\prime} \notin \xi^{+}$. In the following, we consider only non trivial assets, that is each asset has a non zero return in at least one node.

$z=\left(z^{j}\right)_{j \in \mathcal{J}} \in \mathbb{R}^{\mathcal{J}}$ is called the portfolio of agent $i$. If $z^{j}>0$ [resp. $z^{j}<0$ ], then $\left|z^{j}\right|$ is the quantity of asset $j$ bought [resp. sold] by agent $i$ at the issuance node $\xi(j)$.

To summarize a financial structure $\mathcal{F}=\left(\mathcal{J},(\xi(j))_{j \in \mathcal{J}}, V\right)$ consists of

- a set of non trivial assets $\mathcal{J}$,

- a node of issuance $\xi(j)$ for each asset $j \in \mathcal{J}$,

- a payoff mapping $V: \mathbb{R}^{\mathbb{L}} \rightarrow \mathbb{R}^{\mathbb{D} \times \mathcal{J}}$ which associates to every spot price $p \in \mathbb{R}^{\mathbb{L}}$ the $(\mathbb{D} \times \mathcal{J})$-payoff matrix $V(p)=\left(V_{\xi}^{j}(p)\right)_{\xi \in \mathbb{D}, j \in \mathcal{J}}$ and satisfies the condition $V_{\xi}^{j}(p)=0$ if $\xi \notin \mathbb{D}^{+}(\xi(j))$.

The price of asset $j$ is denoted by $q_{j}$; it is paid at its issuance node $\xi(j)$. We let $q=\left(q_{j}\right)_{j \in \mathcal{J}} \in \mathbb{R}^{\mathcal{J}}$ be the asset price vector.

The full payoff matrix $W(p, q)$ is the $(\mathbb{D} \times \mathcal{J})$-matrix with the following entries:

$$
W_{\xi}^{j}(p, q):=V_{\xi}^{j}(p)-\delta_{\xi, \xi(j)} q_{j},
$$

where $\delta_{\xi, \xi^{\prime}}=1$ if $\xi=\xi^{\prime}$ and $\delta_{\xi, \xi^{\prime}}=0$ otherwise.

So, given the prices $(p, q)$, the full flow of returns for a given portfolio $z \in \mathbb{R}^{\mathcal{J}}$ is $W(p, q) z$ and the full return at node $\xi$ is

$$
\begin{gathered}
{[W(p, q) z](\xi):=W_{\xi}(p, q) \bullet \mathcal{J} z=\sum_{j \in \mathcal{J}} V_{\xi}^{j}(p) z^{j}-\sum_{j \in \mathcal{J}} \delta_{\xi, \xi(j)} q_{j} z^{j}} \\
=\sum_{\{j \in \mathcal{J} \backslash \xi(j)<\xi\}} V_{\xi}^{j}(p) z^{j}-\sum_{\{j \in \mathcal{J} \backslash \xi(j)=\xi\}} q_{j} z^{j},
\end{gathered}
$$

We now recall that for a given spot price $p$, the asset price $q$ is an arbitrage free price if it does not exist a portfolio $z \in \mathbb{R}^{\mathcal{J}}$ such that $W(p, q) z>0 . q$ is an arbitrage free price if and only if it exists a so-called state price vector $\lambda \in \mathbb{R}_{++}^{\mathbb{D}}$ such that ${ }^{t} W(p, q) \lambda=0$ (see, e.g. Magill-Quinzii [9]). Taken into account the particular structure of the matrix $W(p, q)$, this is equivalent to

$$
\forall j \in \mathcal{J}, \lambda_{\xi(j)} q_{j}=\sum_{\xi \in \mathbb{D}^{+}(\xi(j))} \lambda_{\xi} V_{\xi}^{j}(p) .
$$

Conversely, for a given state price vector $\lambda \in \mathbb{R}_{++}^{\mathbb{D}}$, there exists a unique associated arbitrage free price denoted $q(\lambda)$ satisfying ${ }^{t} W(p, q) \lambda=0$, which is defined by the above formula.

\section{Some additional notations}


We now introduce some additional notations. For all $\xi \in \mathbb{D} \backslash \mathbb{D}_{T}, \mathcal{J}(\xi)$ is the set of assets issued at the node $\xi$, that is $\mathcal{J}(\xi)=\{j \in \mathcal{J} \mid \xi(j)=\xi\}$ and $\mathcal{J}\left(\mathbb{D}^{-}(\xi)\right)$ is the set of assets issued at a predecessor of $\xi$, that is $\mathcal{J}\left(\mathbb{D}^{-}(\xi)\right)=$ $\{j \in \mathcal{J} \mid \xi(j)<\xi\}$. $\mathbb{D}^{e}$ is the set of nodes at which there is the issuance of at least one asset, that is, $\xi \in \mathbb{D}^{e}$ if $\mathcal{J}(\xi) \neq \emptyset$. If $\xi \notin \mathbb{D}^{e}, \mathcal{J}(\xi)=\emptyset$ and, by convention, we let $\operatorname{Im} V^{\mathcal{J}(\xi)}(p)=\{0\}$.

In all our numerical examples, we assume that there is a unique good at each node of the tree and the price of one unit of the good is equal to 1. Consequently, we will denote the payoff matrix (resp. the full payoff matrix) by $V(\operatorname{resp} . W(q))$.

\subsection{The stochastic exchange economy}

We consider a finite set of consumers $\mathcal{I}=\{1, \ldots, I\}$. Each agent $i \in \mathcal{I}$ has a consumption set $X_{i} \subset \mathbb{R}^{\mathbb{L}}$, which consists of all possible consumptions. An allocation is an element $x \in \prod_{i \in \mathcal{I}} X_{i}$ and we denote by $x_{i}$ the consumption of agent $i$, which is the projection of $x$ on $X_{i}$.

The tastes of each consumer $i \in \mathcal{I}$ are represented by a strict preference correspondence $P_{i}: \prod_{j \in \mathcal{I}} X_{j} \longrightarrow X_{i}$, where $P_{i}(x)$ defines the set of consumptions that are strictly preferred to $x_{i}$ for agent $i$, given the consumption $x_{j}$ for the other consumers $j \neq i$. $P_{i}$ represents the consumer tastes, but also his behavior with respect to time and uncertainty, especially his impatience and attitude toward risk. If consumer preferences are represented by utility functions $u_{i}: X_{i} \longrightarrow \mathbb{R}$ for each $i \in \mathcal{I}$, the strict preference correspondence is defined by $P_{i}(x)=\left\{\bar{x}_{i} \in X_{i} \mid u_{i}\left(\bar{x}_{i}\right)>u_{i}\left(x_{i}\right)\right\}$.

Finally, for each node $\xi \in \mathbb{D}$, every consumer $i \in \mathcal{I}$ has a node endowment $e_{i}(\xi) \in \mathbb{R}^{\mathbb{H}}$ (contingent on the fact that $\xi$ prevails) and we denote by $e_{i}=$ $\left(e_{i}(\xi)\right)_{\xi \in \mathbb{D}} \in \mathbb{R}^{\mathbb{L}}$ the endowments for the whole set of nodes. The exchange economy $\Sigma$ can be summarized by

$$
\Sigma=\left[\mathbb{D}, \mathbb{H}, \mathcal{I},\left(X_{i}, P_{i}, e_{i}\right)_{i \in \mathcal{I}}\right]
$$

\subsection{Financial equilibrium}

We now consider a financial exchange economy, which is defined as the couple of an exchange economy $\Sigma$ and a financial structure $\mathcal{F}$. It can thus be summarized by

$$
(\Sigma, \mathcal{F}):=\left[\mathbb{D}, \mathbb{H}, \mathcal{I},\left(X_{i}, P_{i}, e_{i}\right)_{i \in \mathcal{I}}, \mathcal{J},(\xi(j))_{j \in \mathcal{J}}, V\right] .
$$

Given the price $(p, q) \in \mathbb{R}^{\mathbb{L}} \times \mathbb{R}^{\mathcal{J}}$, the budget set of consumer $i \in \mathcal{I}$ is $B_{\mathcal{F}}^{i}(p, q)$ defined $b y^{2}$ :

$$
\left\{\left(x_{i}, z_{i}\right) \in X_{i} \times \mathbb{R}^{\mathcal{J}}: \forall \xi \in \mathbb{D}, p(\xi) \bullet_{\mathbb{H}}\left[x_{i}(\xi)-e_{i}(\xi)\right] \leq W_{\xi}(p, q) \bullet \mathcal{J} z_{i}\right\}
$$

or

$$
\left\{\left(x_{i}, z_{i}\right) \in X_{i} \times \mathbb{R}^{\mathcal{J}}: p_{\square}\left(x_{i}-e_{i}\right) \leq W(p, q) z_{i}\right\} .
$$

We now introduce the definition of a financial equilibrium:

\footnotetext{
${ }^{2}$ For $x=(x(\xi))_{\xi \in \mathbb{D}}, p=(p(\xi))_{\xi \in \mathbb{D}}$ in $\mathbb{R}^{\mathbb{L}}=\mathbb{R}^{\mathbb{H} \times \mathbb{D}}$ (with $x(\xi), p(\xi)$ in $\mathbb{R}^{\mathbb{H}}$ ) we let $p \square x=$ $(p(\xi) \bullet \mathbb{H} x(\xi))_{\xi \in \mathbb{D}} \in \mathbb{R}^{\mathbb{D}}$.
} 
Definition 2.1. An equilibrium of the financial exchange economy $(\Sigma, \mathcal{F})$ is a list of strategies and prices $(\bar{x}, \bar{z}, \bar{p}, \bar{q}) \in\left(\mathbb{R}^{\mathbb{L}}\right)^{\mathcal{I}} \times\left(\mathbb{R}^{\mathcal{J}}\right)^{\mathcal{I}} \times \mathbb{R}^{\mathbb{L}} \backslash\{0\} \times \mathbb{R}^{\mathcal{J}}$ such that

(a) for every $i \in \mathcal{I},\left(\bar{x}_{i}, \bar{z}_{i}\right)$ maximizes the preferences $P_{i}$ in the budget set $B_{\mathcal{F}}^{i}(\bar{p}, \bar{q})$, in the sense that

$$
\left(\bar{x}_{i}, \bar{z}_{i}\right) \in B_{\mathcal{F}}^{i}(\bar{p}, \bar{q}) \text { and }\left[P_{i}(\bar{x}) \times \mathbb{R}^{\mathcal{J}}\right] \bigcap B_{\mathcal{F}}^{i}(\bar{p}, \bar{q})=\emptyset ;
$$

(b) $\sum_{i \in \mathcal{I}} \bar{x}_{i}=\sum_{i \in \mathcal{I}} e_{i}$ and $\sum_{i \in \mathcal{I}} \bar{z}_{i}=0$.

We recall that the equilibrium asset price is arbitrage free under the following Non-Satiation Assumption:

Assumption NS (i) (Non-Saturation at Every Node) For all $\bar{x} \in \prod_{i \in \mathcal{I}} X_{i}$ such that $\sum_{i \in \mathcal{I}} \bar{x}_{i}=\sum_{i \in \mathcal{I}} e_{i}$, for every $i \in \mathcal{I}$, for every $\xi \in \mathbb{D}$, there exists $x_{i} \in X_{i}$ such that, for each $\xi^{\prime} \neq \xi, x_{i}\left(\xi^{\prime}\right)=\bar{x}_{i}\left(\xi^{\prime}\right)$ and $x_{i} \in P_{i}(\bar{x})$.

(ii) if $x_{i} \in P_{i}(\bar{x})$, then $\left[x_{i}, \bar{x}_{i}\left[\in P_{i}(\bar{x})\right.\right.$.

Proposition 2.1. (Magill-Quinzii [9], Angeloni-Cornet [1]) Under Assumption $(\boldsymbol{N S})$, if $(\bar{x}, \bar{z}, \bar{p}, \bar{q})$ is an equilibrium of the economy $(\Sigma, \mathcal{F})$ then the asset price $\bar{q}$ is arbitrage free i.e., there exists a state price $\lambda \in \mathbb{R}_{++}^{\mathbb{D}}$ such that ${ }^{t} W(\bar{p}, \bar{q}) \lambda=0$.

\section{Equivalent financial structures}

In this section we will define an equivalence relation on financial structures. We will show that the existence of an equilibrium in an exchange economy associated with a given financial structure is equivalent to the existence of equilibrium in exchange economy associated with any other financial structure equivalent to the first one. So equivalence allows to extend the existence results for financial equilibrium to a whole class of financial structures. Hence the importance of studying the notion of equivalence between the financial structures.

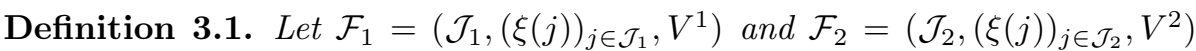
be two financial structures. We say that $\mathcal{F}_{1}$ is equivalent to $\mathcal{F}_{2}$ with respect to a given spot price $p$ (denoted by $\mathcal{F}_{1} \simeq_{p} \mathcal{F}_{2}$ ) if for all state price $\lambda=\left(\lambda_{\xi}\right)_{\xi \in \mathbb{D}} \in$ $\mathbb{R}_{++}^{\mathbb{D}}, \operatorname{Im} W^{1}\left(p, q^{1}(\lambda)\right)=\operatorname{Im} W^{2}\left(p, q^{2}(\lambda)\right)$ where $q^{1}(\lambda)$ and $q^{2}(\lambda)$ are the unique arbitrage free prices associated with $\lambda$.

We say that $\mathcal{F}_{1}$ is equivalent to the $\mathcal{F}_{2}$ if for all spot price vector $p \in \mathbb{R}^{\mathbb{L}}$, $\mathcal{F}_{1} \simeq_{p} \mathcal{F}_{2}$.

The intuition behind this definition is that the financial structures allow agents to transfer wealth across nodes of the date-event tree. Thereby given a spot price $p$, their budget set is determined by the set of marketable payoffs that is the range of the full payoff matrix. To be equivalent, two financial structures must provide the same set of marketable payoffs whatever is the state price and the associated arbitrage free asset prices.

Proposition 3.1. For each spot price $p \in \mathbb{R}^{\mathbb{L}}$, the relation $\simeq_{p}$ defined above is an equivalence relation. 
The proof is left to the reader.

The main consequence of this definition is given below and states that, regardless of the standard exchange economy $\Sigma$, consumption equilibria are the same when agents carry out their financial activities through two different equivalent structures $\mathcal{F}_{1}$ and $\mathcal{F}_{2}$.

Proposition 3.2. Let $\Sigma$ be an exchange economy satisfying Assumption NS. Let $\mathcal{F}_{1}=\left(\mathcal{J}_{1},(\xi(j))_{j \in \mathcal{J}_{1}}, V^{1}\right)$ and $\mathcal{F}_{2}=\left(\mathcal{J}_{2},(\xi(j))_{j \in \mathcal{J}_{2}}, V^{2}\right)$ be two equivalent financial structures.

Let $\left(\bar{x}, \bar{z}, \bar{p}, \bar{q}^{1}\right)$ be an equilibrium of $\left(\Sigma, \mathcal{F}_{1}\right)$. Then there exists $\hat{z}$ and $\bar{q}^{2}$ such that $\left(\bar{x}, \hat{z}, \bar{p}, \bar{q}^{2}\right)$ is an equilibrium of $\left(\Sigma, \mathcal{F}_{2}\right)$.

Proof of Proposition 3.2. Since $\left(\bar{x}, \bar{z}, \bar{p}, \bar{q}^{1}\right)$ is an equilibrium, Proposition 2.1 implies that $\bar{q}^{1}$ is an arbitrage free price. So, there exists a state price $\lambda=$ $\left(\lambda_{\xi}\right)_{\xi \in \mathbb{D}} \in \mathbb{R}_{++}^{\mathbb{D}}$ such that ${ }^{t} W^{1}\left(\bar{p}, \bar{q}^{1}\right) \lambda=0$. Let $\bar{q}^{2}$ be the unique arbitrage free price for the financial structure $\mathcal{F}_{2}$ associed with $\lambda$. Since $\mathcal{F}_{1} \simeq \mathcal{F}_{2}$, we have $\operatorname{Im} W^{1}\left(\bar{p}, \bar{q}^{1}\right)=\operatorname{Im} W^{2}\left(\bar{p}, \bar{q}^{2}\right)$ (See Definition 3.1).

For all $i \neq 1$, let $\hat{z} \in \mathbb{R}^{\mathcal{J}_{2} \mathcal{I}}$ be such that $W^{1}\left(\bar{p}, \bar{q}^{1}\right) \bar{z}_{i}=W^{2}\left(\bar{p}, \bar{q}^{2}\right) \hat{z}_{i}$. Such $\hat{z}_{i}$ exists because $\operatorname{Im} W^{1}\left(\bar{p}, \bar{q}^{1}\right)=\operatorname{Im} W^{2}\left(\bar{p}, \bar{q}^{2}\right)$. Let $\hat{z}_{1}=-\sum_{i \in \mathcal{I} i i \neq 1} \hat{z}_{i}$. We now show that $\left(\bar{x}, \hat{z}, \bar{p}, \bar{q}^{2}\right)$ is an equilibrium of $\left(\Sigma, \mathcal{F}_{2}\right)$. Indeed, for all $i \in \mathcal{I}$,

$$
W^{2}\left(\bar{p}, \bar{q}^{2}\right) \hat{z}_{i}=W^{1}\left(\bar{p}, \bar{q}^{1}\right) \bar{z}_{i}
$$

This is obvious for $i \neq 1$ and if $i=1$, as $\sum_{i \in \mathcal{I}} \bar{z}_{i}=0$,

$$
\begin{gathered}
W^{2}\left(\bar{p}, \bar{q}^{2}\right) \hat{z}_{1}=W^{2}\left(\bar{p}, \bar{q}^{2}\right)\left(-\sum_{i \in \mathcal{I} ; i \neq 1} \hat{z}_{i}\right)=-\sum_{i \in \mathcal{I}_{;} i \neq 1}\left[W^{2}\left(\bar{p}, \bar{q}^{2}\right) \hat{z}_{i}\right]= \\
-\sum_{i \in \mathcal{I} ; i \neq 1}\left[W^{1}\left(\bar{p}, \bar{q}^{1}\right) \bar{z}_{i}\right]=W^{1}\left(\bar{p}, \bar{q}^{1}\right)\left(-\sum_{i \in \mathcal{I} ; i \neq 1} \bar{z}_{i}\right)=W^{1}\left(\bar{p}, \bar{q}^{1}\right) \bar{z}_{1}
\end{gathered}
$$

With this remark, we easily prove that $B_{\mathcal{F}_{1}}^{i}\left(\bar{p}, \bar{q}^{1}\right)=B_{\mathcal{F}_{2}}^{i}\left(\bar{p}, \bar{q}^{2}\right)$ and $\left(\bar{x}_{i}, \hat{z}_{i}\right) \in$ $B_{\mathcal{F}_{2}}^{i}\left(\bar{p}, \bar{q}^{2}\right)$ all $i$, which is enough to conclude since the feasibility conditions are satisfied.

\subsection{Examples of equivalent financial stuctures}

We will give some examples of equivalent financial structures. The proofs are given in Appendix.

Example 3.1. ( Scalar multiplicator) Let $\mathcal{F}=\left(\mathcal{J},(\xi(j))_{j \in \mathcal{J}}, V\right)$ be a financial structure. For each $\alpha \in \mathbb{R} \backslash\{0\}$, the $\alpha$-product of $\mathcal{F}$,

$$
\mathcal{F}_{\alpha}=\left(\mathcal{J},(\xi(j))_{j \in \mathcal{J}}, V^{\alpha}=\alpha V\right)
$$

is equivalent to $\mathcal{F}$. 


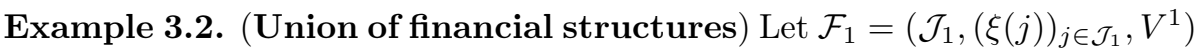
and $\mathcal{F}_{2}=\left(\mathcal{J}_{2},(\xi(j))_{j \in \mathcal{J}_{2}}, V^{2}\right)$ be two financial structures. The financial structure $^{3} \mathcal{F}:=\mathcal{F}_{1} \cup \mathcal{F}_{2}:=\left(\mathcal{J}:=\mathcal{J}_{1} \sqcup \mathcal{J}_{2},(\xi(j))_{j \in \mathcal{J}}, V=\left[V^{1}, V^{2}\right]\right)$ is called the Union of $\mathcal{F}_{1}$ and $\mathcal{F}_{2}, . \mathcal{F}_{1} \cup \mathcal{F}_{2} \simeq \mathcal{F}_{2} \cup \mathcal{F}_{1}$ and if $\mathcal{F}_{1} \simeq_{p} \mathcal{F}_{2}$, then $\mathcal{F}_{1} \cup \mathcal{F}_{2} \simeq_{p} \mathcal{F}_{1}$.

Combining the two previous examples we obtain :

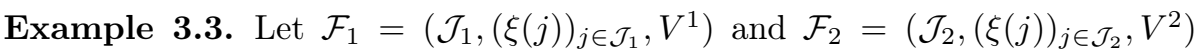
be two financial structures such that $\mathcal{F}_{1} \simeq_{p} \mathcal{F}_{2}$ with respect to the spot price vector $p$. For each pair $(\alpha, \beta) \in \mathbb{R}^{*} \times \mathbb{R}^{*}$, the structure $\mathcal{F}_{\alpha, \beta}:=\alpha \mathcal{F}_{1} \cup \beta \mathcal{F}_{2}=$ $\left(\mathcal{J}=\mathcal{J}_{1} \sqcup \mathcal{J}_{2},(\xi(j))_{j \in \mathcal{J}}, V^{\alpha, \beta}=\left[\alpha V^{1}, \beta V^{2}\right]\right)$ is equivalent to $\mathcal{F}_{1}$ and to $\mathcal{F}_{2}$ with respect to $p$.

Example 3.4. (Stability of the equivalence by reunion.) Let $\mathcal{F}_{1}, \mathcal{F}_{2}, \mathcal{F}_{3}, \mathcal{F}_{4}$ be four financial structures. Let a spot price $p \in \mathbb{R}^{\mathbb{L}}$, such that $\mathcal{F}_{1} \simeq_{p} \mathcal{F}_{2}$ and $\mathcal{F}_{3} \simeq_{p} \mathcal{F}_{4}$ then

$$
\mathcal{F}_{1} \cup \mathcal{F}_{3} \simeq_{p} \mathcal{F}_{2} \cup \mathcal{F}_{4}
$$

Definition 3.2. Let $\mathcal{F}=\left(\mathcal{J},(\xi(j))_{j \in \mathcal{J}}, V\right)$ be a financial structure. We call sub-structure of $\mathcal{F}$ any financial structure $\mathcal{F}^{\prime}=\left(\mathcal{J}^{\prime},(\xi(j))_{j \in \mathcal{J}^{\prime}}, V^{\prime}\right)$ such that $\mathcal{J}^{\prime} \subset \mathcal{J}$ and $V^{\prime}=V^{\mathcal{J}^{\prime}}$

The following proposition is a consequence of Example 3.4.

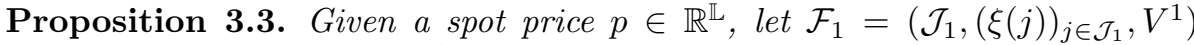
and $\mathcal{F}_{2}=\left(\mathcal{J}_{2},(\xi(j))_{j \in \mathcal{J}_{2}}, V^{2}\right)$ be two financial structures such that there is a sub-structure $\mathcal{F}_{3}$ of $\mathcal{F}_{2}$ which is equivalent to $\mathcal{F}_{1}$ with respect to a spot price $p$. Then we can complete the structure $\mathcal{F}_{1}$ to get a new financial structure $\mathcal{F}$ such that $\mathcal{F} \simeq_{p} \mathcal{F}_{2}$.

\section{Sufficient conditions for equivalence}

In this section we provide sufficient conditions on the payoff matrices for the equivalence of financial structure with long-term assets. In the two-period case, two financial structures are equivalent if the images of their payoff matrices are equal,(see [8]). In the multi-period case, if all assets are short-term, we have generalized this result in [6].

In the multi-period case, if there are long-term assets, the equivalence between two financial structure does not imply that the images of payoff matrices of two financial structures are equal (see below Remark 4.1) and equality between the images of payoff matrices of two financial structures does not imply that these two financial structures are equivalent (see below Remark 4.2).

Remark 4.1. Let $\mathbb{D}=\left\{\xi_{0}, \xi_{1}, \xi_{2}, \xi_{3}\right\}, \mathcal{J}_{1}=\left\{j_{1}^{1}, j_{1}^{2}, j_{1}^{3}\right\}, \mathcal{J}_{2}=\left\{j_{2}^{1}, j_{2}^{2}, j_{2}^{3}\right\}$. $\xi\left(j_{1}^{1}\right)=\xi\left(j_{2}^{1}\right)=\xi_{0}, \xi\left(j_{1}^{2}\right)=\xi\left(j_{2}^{2}\right)=\xi_{1}$ and $\xi\left(j_{1}^{3}\right)=\xi\left(j_{2}^{3}\right)=\xi_{2}$. Let $\lambda=$ $\left(\lambda_{0}, \lambda_{1}, \lambda_{2}, \lambda_{3}\right) \in \mathbb{R}_{++}^{4}$ be a state price and $\left(q_{1}, q_{2}\right) \in \mathbb{R}^{\mathcal{J}_{1}} \times \mathbb{R}^{\mathcal{J}_{2}}$ be the couple of arbitrage free prices for the two financial structures associated to $\lambda$.

\footnotetext{
${ }^{3} \mathcal{J}_{1} \sqcup \mathcal{J}_{2}$ is the union of assets of $\mathcal{F}_{1}$ and of $\mathcal{F}_{2}$ where, the common assets in $\mathcal{J}_{1} \cap \mathcal{J}_{2}$ are counted twice in the new structure, if $\mathcal{J}_{1} \cap \mathcal{J}_{2} \neq \emptyset$. The matrix $\left[V^{1}, V^{2}\right]$ is the $\left(\mathbb{D} \times\left(\mathcal{J}_{1} \sqcup \mathcal{J}_{2}\right)\right)$ matrix whose first $\mathcal{J}_{1}$ columns are those of $V^{1}$ and the last $\mathcal{J}_{2}$ columns are those of $V^{2}$.
} 


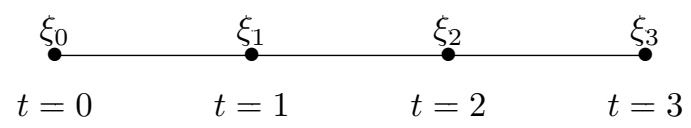

Figure 2: the tree $\mathbb{D}$

The payoff matrices and the full payoff matrices are:

$$
\begin{aligned}
V^{1} & =\left(\begin{array}{lll}
0 & 0 & 0 \\
1 & 0 & 0 \\
1 & 1 & 0 \\
1 & 1 & 1
\end{array}\right) \begin{array}{l}
\xi_{0} \\
\xi_{1} \\
\xi_{2} \\
\xi_{3}
\end{array} \text { and } W^{1}\left(q^{1}\right)=\left(\begin{array}{ccc}
-\frac{\lambda_{1}+\lambda_{2}+\lambda_{3}}{\lambda_{0}} & 0 & 0 \\
1 & -\frac{\lambda_{2}+\lambda_{3}}{\lambda_{1}} & 0 \\
1 & 1 & \frac{-\lambda_{3}}{\lambda_{2}} \\
1 & 1 & 1
\end{array}\right) \begin{array}{l}
\xi_{0} \\
\xi_{1} \\
\xi_{2} \\
\xi_{3}
\end{array} \\
V^{2} & =\left(\begin{array}{lll}
0 & 0 & 0 \\
1 & 0 & 0 \\
1 & 0 & 0 \\
1 & 1 & 1
\end{array}\right) \begin{array}{l}
\xi_{0} \\
\xi_{1} \\
\xi_{2} \\
\xi_{3}
\end{array} \text { and } W^{2}\left(q^{2}\right)=\left(\begin{array}{ccc}
-\frac{\lambda_{1}+\lambda_{2}+\lambda_{3}}{\lambda_{0}} & 0 & 0 \\
1 & -\frac{\lambda_{3}}{\lambda_{1}} & 0 \\
1 & 0 & -\frac{\lambda_{3}}{\lambda_{2}} \\
1 & 1 & 1
\end{array}\right) \begin{array}{l}
\xi_{0} \\
\xi_{1} \\
\xi_{2} \\
\xi_{3}
\end{array}
\end{aligned}
$$

It is clear that $\operatorname{rank} W^{1}\left(q^{1}\right)=\operatorname{rank} W^{2}\left(q^{2}\right)=3$, so $\operatorname{Im} W^{2}\left(q^{2}\right)=\operatorname{Im} W^{1}\left(q^{1}\right)=$ $\lambda^{\perp}$. So the two structures are equivalent although $\operatorname{Im} V^{1} \neq \operatorname{Im} V^{2}$ because $\operatorname{rank} V^{1}=3 \neq \operatorname{rank} V^{2}=2$.

Remark 4.2. Consider two financial structures such that each contains three assets and $\mathbb{D}=\left\{\xi_{)}, \xi_{1}, \xi_{2}, \xi_{11}, \xi_{12}, \xi_{13}, \xi_{21}\right\} \cdot \xi\left(j_{1}^{1}\right)=\xi\left(j_{2}^{1}\right)=\xi\left(j_{2}^{2}\right)=\xi_{0}$ and $\xi\left(j_{1}^{2}\right)=\xi\left(j_{1}^{3}\right)=\xi\left(j_{2}^{3}\right)=\xi_{1}$.

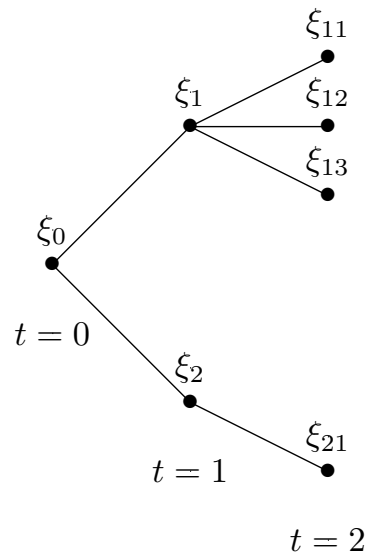

Figure 3: the tree $\mathbb{D}$

The two payoff matrices $V^{1}$ and $V^{2}$ are equal, so they have the same image 


$$
V^{1}=V^{2}=\left(\begin{array}{ccc|c}
0 & 0 & 0 \\
1 & 0 & 0 & \xi_{0} \\
1 & 0 & 0 & \xi_{1} \\
0 & 0 & 1 & \xi_{11} \\
0 & 1 & 0 & \xi_{12} \\
1 & 0 & 0 & \xi_{13} \\
1 & 0 & 0 & \xi_{21}
\end{array}\right.
$$

With $\lambda=(1,1,1,1,1,1,1)$ and $q^{1}$ and $q^{2}$ the two associated arbitrage free prices, we have:

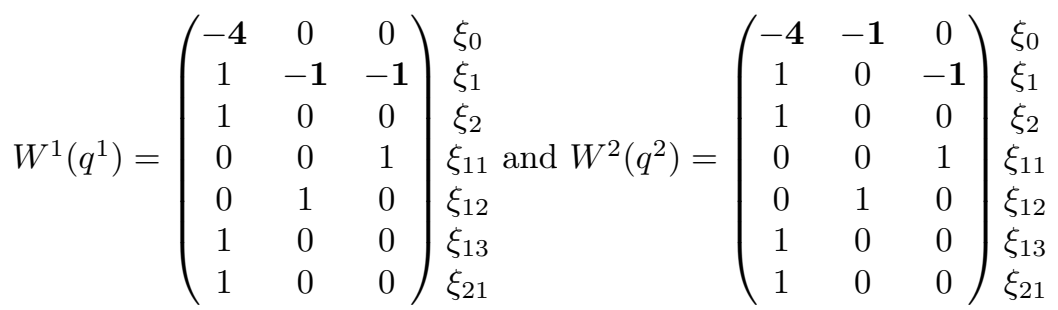

The two structures are not equivalent since $\operatorname{Im} W^{1}\left(q^{1}\right) \neq \operatorname{Im} W^{2}\left(q^{2}\right)$ even if the two payoff matrices have the same image. Indeed, we can check that the second column vector of the matrix $W^{2}\left(q^{2}\right)$ does not belong to $\operatorname{Im} W^{1}\left(q^{1}\right)$.

\subsection{Equivalence under Assumption R1}

To get the equivalence, we need an additional assumption on the payoff matrices that we now introduce:

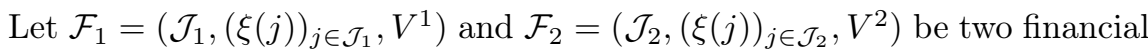
structures defined on the same date-event tree $\mathbb{D}$ and $p \in \mathbb{R}^{\mathbb{L}}$ be a spot price vector.

Assumption R1. $\forall \xi \in \mathbb{D}_{1}^{e} \cup \mathbb{D}_{2}^{e}$,

$$
\operatorname{Im} V^{1 \mathcal{J}_{1}(\xi)}(p)=\operatorname{Im} V^{2 \mathcal{J}_{2}(\xi)}(p) .
$$

Assumption R1 means that at each emission node $\xi$, both structures offer the same possibilities of transfer between successor nodes to $\xi$. In the two-period case, Assumption R1 at $p$ simply means $\operatorname{Im} V^{1}(p)=\operatorname{Im} V^{2}(p)$ since there is only

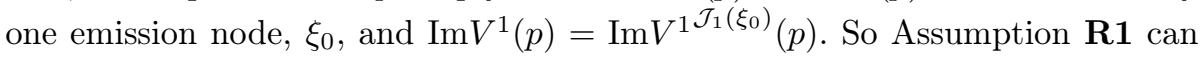
be seen as the natural extension of the standard assumption on the equality of the range of the payoff matrices when there are more than one issuance node. We provide additional comments on Assumption R1 in Sub-section 4.2.

Note that since trivial assets are excluded, if Assumption R1 is satisfied, then the issuance nodes are the same for both financial structures.

We now state the main result of this paper on equivalence with long-term assets when markets are incomplete or not.

Proposition 4.1. Given a spot price vector $p \in \mathbb{R}^{\mathbb{L}}$. Let $\mathcal{F}_{1}$ and $\mathcal{F}_{2}$ be two financial structures satisfying Assumption $\mathbf{R} 1$ at the spot price $p \in \mathbb{R}^{\mathbb{L}}$. Then $\mathcal{F}_{1} \simeq_{p} \mathcal{F}_{2}$. 
Proof of Proposition 4.1. Since

$$
\operatorname{Im} V^{1}(p)=\sum_{\xi \in \mathbb{D}_{1}^{e}} \operatorname{Im} V^{\mathcal{J}_{1}(\xi)}(p) \text { and } \operatorname{Im} V^{2}(p)=\sum_{\xi \in \mathbb{D}_{2}^{e}} \operatorname{Im} V^{2 \mathcal{J}_{2}(\xi)}(p)
$$

and since Assumption $\mathbf{R} \mathbf{1}$ implies that $\mathbb{D}_{1}^{e}=\mathbb{D}_{2}^{e}=\mathbb{D}^{e}$, one concludes that $\operatorname{Im} V^{1}(p)=\operatorname{Im} V^{2}(p)$ under Assumption R1.

Conversely, let $\lambda \in \mathbb{R}_{++}^{\mathbb{D}}$ be a state price and let $q^{1}$ and $q^{2}$ be the two associated arbitrage free prices. Let $y \in \operatorname{Im} W^{1}\left(p, q^{1}\right)$. There exists $z^{1} \in \mathbb{R}^{\mathcal{J}_{1}}$ such that

$$
y=W^{1}\left(p, q^{1}\right) z^{1}=\sum_{\xi \in \mathbb{D}^{e}}\left[\sum_{j \in \mathcal{J}_{1}(\xi)} W^{1, j}\left(p, q^{1}\right) z^{1, j}\right] .
$$

Let $\xi \in \mathbb{D}$ be given. We have:

$$
\sum_{j \in \mathcal{J}_{1}} W_{\xi}^{1, j}\left(p, q^{1}\right) z^{1, j}=\left\{\begin{array}{l}
\sum_{j \in \mathcal{J}_{1}} V_{\xi}^{1, j}(p) z^{1, j} \text { if } \xi \notin \mathbb{D}^{e} \\
\sum_{j \in \mathcal{J}_{1} \backslash \mathcal{J}_{1}(\xi)} V_{\xi}^{1, j}(p) z^{1, j}-\sum_{j \in \mathcal{J}_{1}(\xi)} q_{j}^{1} z^{1, j} \text { if } \xi \in \mathbb{D}^{e}
\end{array}\right.
$$

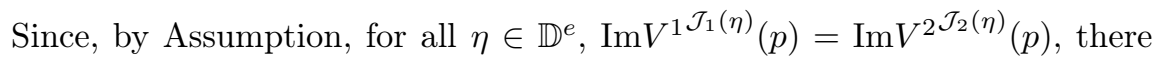
exists $z^{2} \in \mathbb{R}^{\mathcal{J}_{2}}$ such that, for all $\eta \in \mathbb{D}^{e}$,

$$
\sum_{j \in \mathcal{J}_{1}(\eta)} V^{1, j}(p) z^{1, j}=\sum_{j \in \mathcal{J}_{2}(\eta)} V^{2, j}(p) z^{2, j} .
$$

This implies that

$$
\sum_{j \in \mathcal{J}_{1}} W_{\xi}^{1, j}\left(p, q^{1}\right) z^{1, j}=\left\{\begin{array}{l}
\sum_{j \in \mathcal{J}_{2}} V_{\xi}^{2, j}(p) z^{2, j} \text { if } \xi \notin \mathbb{D}^{e} \\
\sum_{j \in \mathcal{J}_{2} \backslash \mathcal{J}_{2}(\xi)} V_{\xi}^{2, j}(p) z^{2, j}-\sum_{j \in \mathcal{J}_{1}(\xi)} q_{j}^{1} z^{1, j} \text { if } \xi \in \mathbb{D}^{e}
\end{array}\right.
$$

But, since ${ }^{t} W^{1}\left(p, q^{1}\right) \lambda=0$, with $\xi \in \mathbb{D}^{e}$,

$$
\begin{gathered}
\sum_{j \in \mathcal{J}_{1}(\xi)} q_{j}^{1} z^{1, j}=\sum_{j \in \mathcal{J}_{1}(\xi)}\left[\sum_{\xi^{\prime} \in \mathbb{D}^{+}(\xi)} \frac{\lambda_{\xi^{\prime}}}{\lambda_{\xi}} V_{\xi^{\prime}}^{1, j}(p)\right] z^{1, j} \\
=\frac{1}{\lambda_{\xi}} \sum_{\xi^{\prime} \in \mathbb{D}^{+}(\xi)}\left[\lambda_{\xi^{\prime}}\left[\sum_{j \in \mathcal{J}_{1}(\xi)} V_{\xi^{\prime}}^{1, j}(p) z^{1, j}\right]\right] .
\end{gathered}
$$

Since for all $\eta \in \mathbb{D}^{e}, \sum_{j \in \mathcal{J}_{1}(\eta)} V^{1, j}(p) z^{1, j}=\sum_{j \in \mathcal{J}_{2}(\eta)} V^{2, j}(p) z^{2, j}$, for each $\xi^{\prime} \in \mathbb{D}^{+}(\xi)$

$$
\sum_{j \in \mathcal{J}_{1}(\xi)} V_{\xi^{\prime}}^{1, j}(p) z^{1, j}=\sum_{j \in \mathcal{J}_{2}(\xi)} V_{\xi^{\prime}}^{2, j}(p) z^{2, j} .
$$

Consequently, since ${ }^{t} W^{2}\left(p, q^{2}\right) \lambda=0$, 


$$
\begin{aligned}
& \frac{1}{\lambda_{\xi}} \sum_{\xi^{\prime} \in \mathbb{D}^{+}(\xi)}\left[\lambda_{\xi^{\prime}}\left[\sum_{j \in \mathcal{J}_{1}(\xi)} V_{\xi^{\prime}}^{1, j}(p) z^{1, j}\right]\right] \\
= & \frac{1}{\lambda_{\xi}} \sum_{\xi^{\prime} \in \mathbb{D}^{+}(\xi)}\left[\lambda_{\xi^{\prime}}\left[\sum_{j \in \mathcal{J}_{2}(\xi)} V_{\xi^{\prime}}^{2, j}(p) z^{2, j}\right]\right] \\
= & \sum_{j \in \mathcal{J}_{2}(\xi)}\left[\sum_{\xi^{\prime} \in \mathbb{D}^{+}(\xi)} \frac{\lambda_{\xi^{\prime}}}{\lambda_{\xi}} V_{\xi^{\prime}}^{2, j}(p)\right] z^{2, j}=\sum_{j \in \mathcal{J}_{2}(\xi)} q_{j}^{2} z^{2, j} .
\end{aligned}
$$

Hence

$$
\begin{gathered}
\sum_{j \in \mathcal{J}_{1}} W_{\xi}^{1, j}\left(p, q^{1}\right) z^{1, j}=\left\{\begin{array}{l}
\sum_{j \in \mathcal{J}_{2}} V_{\xi}^{2, j}(p) z^{2, j} \text { if } \xi \notin \mathbb{D}^{e} \\
\sum_{j \in \mathcal{J}_{2} \backslash \mathcal{J}_{2}(\xi)} V_{\xi}^{2, j}(p) z^{2, j}-\sum_{j \in \mathcal{J}_{1}(\xi)} q_{j}^{2} z^{2, j} \text { if } \xi \in \mathbb{D}^{e}
\end{array}\right. \\
=\sum_{j \in \mathcal{J}_{2}} W_{\xi}^{2, j}\left(p, q^{2}\right) z^{2, j}
\end{gathered}
$$

So for all $\xi \in \mathbb{D}, y_{\xi}=\sum_{j \in \mathcal{J}_{1}} W_{\xi}^{1, j}\left(p, q^{1}\right) z^{1, j}=\sum_{j \in \mathcal{J}_{2}} W_{\xi}^{2, j}\left(p, q^{2}\right) z^{2, j}$. Consequently $y \in \operatorname{Im} W^{2}\left(p, q^{2}\right)$ hence $\operatorname{Im} W^{1}\left(p, q^{1}\right) \subset \operatorname{Im} W^{2}\left(p, q^{2}\right)$. With a similar reasoning, we can show that $\operatorname{Im} W^{2}\left(p, q^{2}\right) \subset \operatorname{Im} W^{1}\left(p, q^{1}\right)$. So $\operatorname{Im} W^{2}\left(p, q^{2}\right)=$ $\operatorname{Im} W^{1}\left(p, q^{1}\right)$, that is $\mathcal{F}_{1} \simeq_{p} \mathcal{F}_{2}$.

Remark 4.3. Assumption $\mathbf{R} 1$ is not necessary for the equivalence of financial structures. Indeed, we provide now two equivalent financial structures, which do not satisfy Assumption R1. Let $\mathbb{D}=\left\{\xi_{0}, \xi_{1}, \xi_{2}, \xi_{3}\right\}$ as in Remark 4.1, $\mathcal{J}_{1}=$ $\left\{j_{1}^{1}, j_{1}^{2}, j_{1}^{3}\right\}, \mathcal{J}_{2}=\left\{j_{2}^{1}, j_{2}^{2}, j_{2}^{3}\right\} \cdot \xi\left(j_{1}^{1}\right)=\xi\left(j_{1}^{2}\right)=\xi\left(j_{2}^{1}\right)=\xi\left(j_{2}^{2}\right)=\xi_{0}, \xi\left(j_{1}^{3}\right)=\xi_{2}$ and $\xi\left(j_{2}^{3}\right)=\xi_{1}$. Let $\lambda=\left(\lambda_{0}, \lambda_{1}, \lambda_{2}, \lambda_{3}\right)$ be a state price and let $\left(q^{1}, q^{2}\right) \in$ $\mathbb{R}^{\mathcal{J}_{1}} \times \mathbb{R}^{\mathcal{J}_{2}}$ be the couple of associated arbitrage free prices.

The payoff matrices and the full payoff matrices are:

$$
\begin{gathered}
V^{1}=\left(\begin{array}{lll}
0 & 0 & 0 \\
1 & 0 & 0 \\
0 & 1 & 0 \\
0 & 1 & 1
\end{array}\right) \begin{array}{l}
\xi_{0} \\
\xi_{1} \\
\xi_{2} \\
\xi_{3}
\end{array} \text { and } W^{1}\left(q^{1}\right)=\left(\begin{array}{ccc}
-\frac{\lambda_{1}}{\lambda_{0}} & \frac{-\lambda_{2}-\lambda_{3}}{\lambda_{0}} & 0 \\
1 & 0 & 0 \\
0 & 1 & \frac{-\lambda_{3}}{\lambda_{2}} \\
0 & 1 & 1
\end{array}\right) \begin{array}{l}
\xi_{0} \\
\xi_{1} \\
\xi_{2} \\
\xi_{3}
\end{array} \\
V^{2}=\left(\begin{array}{ccc}
0 & 0 & 0 \\
1 & 0 & 0 \\
0 & 1 & -1 \\
1 & -1 & 0
\end{array}\right) \begin{array}{l}
\xi_{0} \\
\xi_{1} \\
\xi_{2} \\
\xi_{3}
\end{array} \text { and } W^{2}\left(q^{2}\right)=\left(\begin{array}{ccc}
\frac{-\lambda_{1}-\lambda_{3}}{\lambda_{0}} & \frac{-\lambda_{2}+\lambda_{3}}{\lambda_{0}} & 0 \\
1 & 0 & \frac{\lambda_{2}}{\lambda_{1}} \\
0 & 1 & -1 \\
1 & -1 & 0
\end{array}\right) \begin{array}{l}
\xi_{0} \\
\xi_{1} \\
\xi_{2} \\
\xi_{3}
\end{array}
\end{gathered}
$$

Assumption R1 is not satisfied since the issuance nodes are not the same for the two financial structures. We have $\operatorname{rank} W^{1}\left(q_{1}\right)=\operatorname{rank} W^{2}\left(q_{2}\right)=3$. Indeed, the rank of the square sub-matrix $A_{1}$ (resp. $A_{2}$ ) composed by the three last 
rows of $W^{1}\left(q^{1}\right)$ (resp. $W^{2}\left(q^{2}\right)$ ) is equal to 3 because $\operatorname{Det} A_{1}=1+\frac{\lambda_{3}}{\lambda_{2}}$ (resp. $\left.\operatorname{Det} A_{2}=-1-\frac{\lambda_{2}}{\lambda_{1}}\right)$ which is always different from zero. So, one can conclude that $\operatorname{Im} W^{1}\left(q^{1}\right)=\operatorname{Im} W^{2}\left(q^{2}\right)=\lambda^{\perp}$, hence the financial structures are equivalent.

\subsection{Comments on Assumption R1}

We first remark that Assumption $\mathbf{R} \mathbf{1}$ is stronger than assuming the equality of the images of the two payoff matrices. Indeed, in Example 4.3, $\operatorname{Im} V^{1}=\operatorname{Im} V^{2}$ but Assumption R1 is not satisfied. The converse is true as already noticed at the beginning of the proof of Proposition 4.1.

With short term assets, Assumption R1 is equivalent to the equality of the images of the payoff matrices.

Lemma 4.1. Let $p \in \mathbb{R}^{\mathbb{L}}$ be a spot price vector. Let $\mathcal{F}_{1}=\left(\mathcal{J}_{1},(\xi(j))_{j \in \mathcal{J}_{1}}, V^{1}\right)$ and $\mathcal{F}_{2}=\left(\mathcal{J}_{2},(\xi(j))_{j \in \mathcal{J}_{2}}, V^{2}\right)$ be two financial structures with only short-term assets. Then $\operatorname{Im} V^{1}(p)=\operatorname{Im} V^{2}(p)$ if and only if Assumption $\mathbf{R} \mathbf{1}$ holds true at the spot price $p$.

The proof of Lemma 4.1 is given in Appendix.

The next proposition provides sufficient conditions under which Assumption R1 holds true. We first recall the assumption introduced by Bonnisseau and Chéry in [7]. Given a spot price vector $p \in \mathbb{R}^{\mathbb{L}}$, and a payoff matrix $V(p)$.

Assumption R. $\forall \xi \in \mathbb{D}^{e}$,

$$
\operatorname{Vect}\left(V_{\mathbb{D}^{+}(\xi)}^{\mathcal{J}\left(\mathbb{D}^{-}(\xi)\right)}(p)\right) \bigcap \operatorname{Vect}\left(V_{\mathbb{D}^{+}(\xi)}^{\mathcal{J}(\xi)}(p)\right)=\{0\} .
$$

Assumption $\mathbf{R}$ means that the returns of the assets issued at a node $\xi$ are not redundant with the returns of the assets issued at a predecessor node of $\xi$. So, the issuance of additional assets at $\xi$ are a true financial innovation since the payoffs in the successors of $\xi$ cannot be replicated by the payoffs of a portfolio built with the assets issued before $\xi$.

Proposition 4.2. Let $p \in \mathbb{R}^{\mathbb{L}}$ be a spot price vector. Let $\mathcal{F}_{1}$ and $\mathcal{F}_{2}$ be two financial structures. They satisfy Assumption $\mathbf{R} 1$ at the spot price $p$ if one of the following conditions is satisfied:

(i) All assets are issued at the same date $\tau_{1}$ and $\operatorname{Im} V^{1}(p)=\operatorname{Im} V^{2}(p)$.

(ii) For all $\xi \in \mathbb{D}_{1}^{e} \cup \mathbb{D}_{2}^{e}, \operatorname{Im} V^{1 \mathcal{J}_{1}(\xi)}(p) \subset \operatorname{Im} V^{2 \mathcal{J}_{2}(\xi)}(p)$, Assumption $\boldsymbol{R}$ is satisfied at the spot price $p$ by both financial structures and $\operatorname{rank} V^{1}(p)=$ $\operatorname{rank} V^{2}(p)$.

The proof is given in Appendix.

We show in the next sub-section that Assumption R1 is satisfied when we consider re-trading extensions of financial structures with emission of primitive assets only at node $\xi_{0}$ and equality of the ranges of the payoff matrices associated to the primitive assets. 


\subsection{Equivalence with re-trading}

With more than two periods, the financial structure is often built upon primitive assets issued at the initial nodes providing payoffs for the future periods and then re-traded at the successive periods. See Magill-Quinzii [9] for a complete description. In Angeloni-Cornet [1], it is shown that the re-trading of an asset can be interpreted as the issuance of a new asset with the payoff being the truncation of the payoffs of the initial asset for the future nodes. They have also proved that we get equivalent financial structures by considering a payoff matrix à la Magill-Quinzii where the asset prices appears two times since the asset can be sold and bought at each non-terminal node or the payoff matrix à la Angeloni-Cornet, where each asset is exchanged only once at the issuance node.

For the sake of simplicity and consistency with the previous sections, we consider the Angeloni-Cornet approach. We show that the equality of the range of the payoff matrices for the primitive assets is sufficient for the equivalence of the re-trading extension.

Let $\mathcal{F}=\left(\mathcal{J},(\xi(j))_{j \in \mathcal{J}}, V\right)$ be a financial structure such that all assets are issued at node $\xi_{0}$. Suppose that each asset $j$, once issued, is re-traded at all succeeding nodes except terminal nodes. Each re-traded asset at a node $\xi \in \mathbb{D}^{-}$ is considered as a new asset $j_{\xi}$ issued at node $\xi$. The new financial structure thus constituted is called the re-trading extension of the primitive financial structure.

Definition 4.1. Let $\mathcal{F}=\left(\mathcal{J},(\xi(j))_{j \in \mathcal{J}}, V\right)$ be a financial structure where $\xi(j)=\xi_{0}$ for all $j$. The re-trading of asset $j \in \mathcal{J}$ at node $\xi^{\prime}$, denoted $j_{\xi^{\prime}}$, is the asset issued at $\xi^{\prime}$, that is, $\xi\left(j_{\xi^{\prime}}\right)=\xi^{\prime}$, and whose flow of payoffs is given by

$$
\begin{aligned}
& \tilde{V}_{\xi}^{j_{\xi^{\prime}}}(p)=V_{\xi}^{j}(p), \text { if } \xi \in \mathbb{D}^{+}\left(\xi^{\prime}\right) ; \\
& \tilde{V}_{\xi}^{j_{\xi^{\prime}}}(p)=0 \text { otherwise. }
\end{aligned}
$$

The re-trading extension of $\mathcal{F}$ is the the new financial structure

$$
\left(\tilde{\mathcal{J}}=\mathcal{J} \times \mathbb{D}^{-},\left(\xi\left(j^{\prime}\right)\right)_{j^{\prime} \in \tilde{\mathcal{J}}}, \tilde{V}\right)
$$

which consists of all primitive assets $j \in \mathcal{J}$ and of all re-trading assets $\left(j_{\xi^{\prime}}\right)$ to all nodes $\xi^{\prime} \in \mathbb{D}^{+}\left(\xi_{0}\right) \backslash\left\{\mathbb{D}_{T}\right\}$.

\section{A simple example}

Let $\mathcal{F}$ be a financial structure constituted of two financial assets $\left\{j^{1}, j^{2}\right\}$ issued at the first date such that

$$
V^{j^{1}}={ }^{t}(1,3,-2,1,1,4) \text { and } V^{j^{2}}={ }^{t}(2,-2,3,-1,1,1)
$$

and $\mathbb{D}=\left\{\xi_{0}, \xi_{1}, \xi_{2}, \xi_{11}, \xi_{12}, \xi_{21}, \xi_{22}\right\}$.

The payoff matrix of the re-trading extension $\tilde{\mathcal{F}}$ of $\mathcal{F}$ is: 


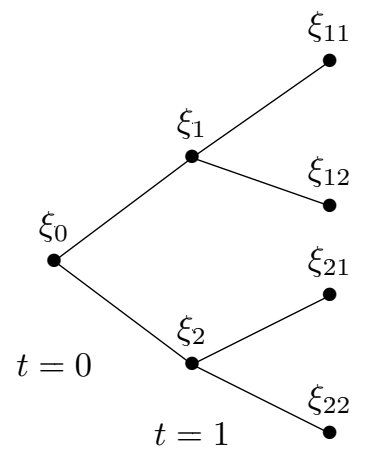

$t=2$

Figure 4: the tree $\mathbb{D}$

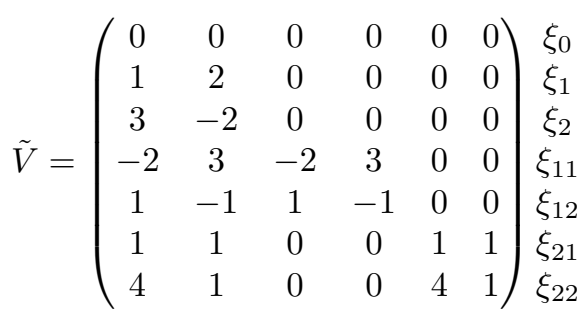

\section{Notations:}

For each $\xi^{\prime} \in \mathbb{D}^{-}$, we denote by $\tilde{q}_{j_{\xi^{\prime}}}$ the price of asset $j_{\xi^{\prime}}$ (i.e., the re-trading of asset $j$ at node $\xi^{\prime}$ ), which is sometimes also called the re-trading price of asset $j$ at node $\xi^{\prime}$. So, for the financial structure $\tilde{\mathcal{F}}$, both the asset price vector $\tilde{q}=\left(\tilde{q}_{\xi_{\xi^{\prime}}}\right)_{j_{\xi^{\prime}} \in \tilde{\mathcal{J}}=\mathcal{J} \times \mathbb{D}^{-}}$and the portfolio $z=\left(z^{j_{\xi^{\prime}}}\right)_{\left(j_{\xi^{\prime}}\right) \in \tilde{\mathcal{J}}=\mathcal{J} \times \mathbb{D}^{-}}$now belong to $\mathbb{R}^{\mathcal{J} \times \mathbb{D}^{-}}$.

Given a spot price $p \in \mathbb{R}^{\mathbb{L}}$ an asset price vector $q \in \mathbb{R}^{\mathcal{J} \times \mathbb{D}^{-}}$and a portfolio $z \in \mathbb{R}^{\mathcal{J} \times \mathbb{D}^{-}}$, the full financial return of $z$ for the financial structure $\tilde{\mathcal{F}}$ at node $\xi \in \mathbb{D}$ is given by:

if $\xi=\xi_{0}, W_{\tilde{\mathcal{F}} \xi}(p, \tilde{q}) \bullet \tilde{\mathcal{J}} z=-\sum_{j_{\xi_{0}} \in \tilde{\mathcal{J}}\left(\xi_{0}\right)=\mathcal{J} \times\left\{\xi_{0}\right\}} \tilde{q}_{j_{\xi_{0}}} z^{j_{\xi_{0}}}$

if $\xi \in \mathbb{D}^{-} \cap \mathbb{D}^{+}, W_{\tilde{\mathcal{F}} \xi}(p, \tilde{q}) \bullet \tilde{\mathcal{J}} z$ is equal to

$$
\begin{aligned}
& \sum_{j_{\xi^{\prime}} \in \tilde{\mathcal{J}}=\mathcal{J} \times \mathbb{D}^{-}} \tilde{V}_{\xi}^{j_{\xi^{\prime}}}(p) z^{j_{\xi^{\prime}}}-\sum_{j_{\xi^{\prime}} \in \tilde{\mathcal{J}}=\mathcal{J} \times \mathbb{D}^{-}} \delta_{\xi, \xi\left(j_{\xi^{\prime}}\right)} \tilde{q}_{j_{\xi^{\prime}}} z^{j_{\xi^{\prime}}} \\
& =\sum_{\left\{j_{\xi^{\prime}} \in \tilde{\mathcal{J}} \mid \xi\left(j_{\xi^{\prime}}\right)<\xi\right\}} \tilde{V}_{\xi}^{j_{\xi^{\prime}}}(p) z^{j_{\xi^{\prime}}}-\sum_{j_{\xi} \in \tilde{\mathcal{J}}(\xi)=\mathcal{J} \times\{\xi\}} \tilde{q}_{j_{\xi}} z^{j_{\xi}} \\
& =\sum_{\xi^{\prime} \in \mathbb{D}^{-}(\xi)}\left(\sum_{j_{\xi^{\prime}} \in \tilde{\mathcal{J}}} \tilde{V}_{\xi}^{j_{\xi^{\prime}}}(p) z^{j_{\xi^{\prime}}}\right)-\sum_{j_{\xi} \in \mathcal{J} \times\{\xi\}} \tilde{q}_{j_{\xi}} z^{j_{\xi}} \\
& =\sum_{\xi^{\prime} \in \mathbb{D}^{-}(\xi)}\left(\sum_{j \in \mathcal{J}} V_{\xi}^{j}(p) z^{j_{\xi^{\prime}}}\right)-\sum_{j_{\xi} \in \mathcal{J} \times\{\xi\}} \tilde{q}_{j_{\xi}} z^{j_{\xi}}
\end{aligned}
$$

finally if $\xi \in \mathbb{D}_{T}, W_{\tilde{\mathcal{F}} \xi}(p, \tilde{q}) \bullet_{\tilde{\mathcal{J}}} z$ is equal to:

$$
\sum_{\xi^{\prime} \in \mathbb{D}^{-}(\xi)}\left(\sum_{j_{\xi^{\prime}} \in \tilde{\mathcal{J}}} \tilde{V}_{\xi}^{j_{\xi^{\prime}}}(p) z^{j_{\xi^{\prime}}}\right)=\sum_{\xi^{\prime} \in \mathbb{D}^{-}(\xi)}\left(\sum_{j \in \mathcal{J}} V_{\xi}^{j}(p) z^{j_{\xi^{\prime}}}\right)
$$


So, the full payoff matrix of the previous example is:

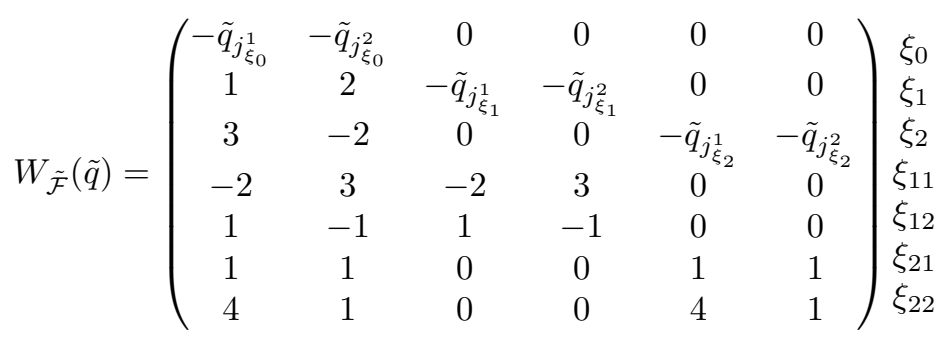

We now state a simple condition under which the re-trading extensions of two primitive financial structures satisfy Assumption R1. Actually, the necessary and sufficient condition is that the ranges of the payoff matrices of the primitive financial structures be equal.

Proposition 4.3. Let $p \in \mathbb{R}^{\mathbb{L}}$ be a spot price. Consider two financial structures $\mathcal{F}_{1}=\left(\mathcal{J}_{1},(\xi(j))_{j \in \mathcal{J}_{1}}, V^{1}\right)$ and $\mathcal{F}_{2}=\left(\mathcal{J}_{2},(\xi(j))_{j \in \mathcal{J}_{2}}, V^{2}\right)$ where all assets are is-

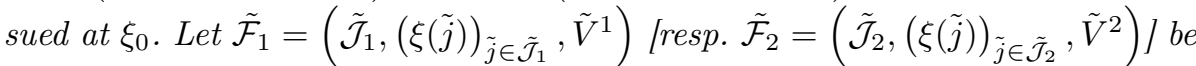
the re-trading extension of $\mathcal{F}_{1}\left[\right.$ resp. $\left.\mathcal{F}_{2}\right]$. The following assertions are equivalent

1) $\mathcal{F}_{1} \simeq_{p} \mathcal{F}_{2}$;

2) $\operatorname{Im} V^{1}(p)=\operatorname{Im} V^{2}(p)$;

3) Assumption $\mathbf{R} \mathbf{1}$ is satisfied with respect to $p$ by the financial structures $\tilde{\mathcal{F}}_{1}$ and $\tilde{\mathcal{F}}_{2}$

Proof of Proposition 4.3. 1) implies 2). Let $\lambda \in \mathbb{R}_{++}^{\mathbb{D}}$ and $\left(q_{1}, q_{2}\right)$ the couple of associated arbitrage free prices. Then, by definition of equivalence between financial structure, $\operatorname{Im} W^{1}\left(p, q_{1}\right)=\operatorname{Im} W^{2}\left(p, q_{2}\right)$. Let $y \in \operatorname{Im} V^{1}(p)$. There exists $z \in \mathbb{R}^{\mathcal{J}_{1}}$ such that $y=V^{1}(p) z$. Since all assets are issued at $\xi_{0},\left(-q_{1} \cdot z, y\right)=W^{1}\left(p, q_{1}\right) z \in \operatorname{Im} W^{1}\left(p, q_{1}\right)$. So, there exists $\zeta \in \mathbb{R}^{\mathcal{J}_{2}}$ so that $\left(-q_{1} \cdot z, y\right)=W^{2}\left(p, q_{2}\right) \zeta=\left(-q_{2} \cdot \zeta, V^{2}(p) \zeta\right)$. Hence, $y=V^{2}(p) \zeta$, so $y \in \operatorname{Im} V^{2}(p)$. Consequently $\operatorname{Im} V^{1}(p) \subset \operatorname{Im} V^{2}(p)$ and one proves the converse inclusion in the same way.

2) implies 3). The matrix $\tilde{V}^{1, \tilde{\mathcal{J}}_{1}(\xi)}(p)$ (resp. $\left.\tilde{V}^{2, \tilde{\mathcal{J}}_{2}(\xi)}(p)\right)$ is obtained from the matrix $V^{1}(p)$ (resp. $V^{2}(p)$ ) by replacing the rows for the nodes $\eta \notin \mathbb{D}^{+}(\xi)$ by rows with zero entries. So, the range of $\tilde{V}^{1, \tilde{\mathcal{J}}_{1}(\xi)}(p)$ (resp. $\left.\tilde{V}^{2, \tilde{\mathcal{J}}_{2}(\xi)}(p)\right)$ is just the projection of the range of $V^{1}(p)$ (resp. $\left.V^{2}(p)\right)$ on the space of vectors in $\mathbb{R}^{\mathbb{D}}$ with zero entries for $\eta \notin \mathbb{D}^{+}(\xi)$. Hence, since the ranges of $V^{1}(p)$ and $V^{2}(p)$ are equal, the ranges of $\tilde{V}^{1, \tilde{\mathcal{J}}_{1}(\xi)}(p)$ and $\tilde{V}^{2, \tilde{\mathcal{J}}_{2}(\xi)}(p)$ are also equal, so Assumption $\mathbf{R} 1$ is satisfied with respect to $p$ by the financial structures $\tilde{\mathcal{F}}_{1}$ and $\tilde{\mathcal{F}}_{2}$.

3) implies 1). Indeed, if Assumption $\mathbf{R} 1$ is satisfied with respect to $p$ by the financial structures $\tilde{\mathcal{F}}_{1}$ and $\tilde{\mathcal{F}}_{2}$, for the node $\xi_{0}, \operatorname{Im} \tilde{V}^{1, \tilde{\mathcal{J}}_{1}\left(\xi_{0}\right)}(p)=\operatorname{Im} \tilde{V}^{2, \mathcal{J}_{2}\left(\xi_{0}\right)}(p)$. Thanks to the definition of a re-trading extension and the fact that all assets are issued at $\xi_{0}$ for the financial structures $\mathcal{F}_{1}$ and $\mathcal{F}_{2}, \tilde{V}^{1, \tilde{\mathcal{J}}_{1}\left(\xi_{0}\right)}(p)=V^{1}(p)$ and $\tilde{V}^{2, \tilde{\mathcal{J}}_{2}\left(\xi_{0}\right)}(p)=V^{2}(p)$. So $\operatorname{Im} V^{1}(p)=\operatorname{Im} V^{2}(p)$.

A direct consequence of the above proposition and Proposition 4.1 is the following sufficient condition for the equivalence of the re-trading extensions of two primitive financial structures. 
Corollary 4.1. Let $p \in \mathbb{R}^{\mathbb{L}}$ be a spot price. Consider two financial structures $\mathcal{F}_{1}=\left(\mathcal{J}_{1},(\xi(j))_{j \in \mathcal{J}_{1}}, V^{1}\right)$ and $\mathcal{F}_{2}=\left(\mathcal{J}_{2},(\xi(j))_{j \in \mathcal{J}_{2}}, V^{2}\right)$ where all assets are issued at $\xi_{0}$. Let $\tilde{\mathcal{F}}_{1}$ [resp. $\tilde{\mathcal{F}}_{2}$ ] be the re-trading extension of $\mathcal{F}_{1}$ [resp. $\mathcal{F}_{2}$ ]. Then $\operatorname{Im} V^{1}(p)=\operatorname{Im} V^{2}(p)$ implies $\tilde{\mathcal{F}}_{1} \simeq_{p} \tilde{\mathcal{F}}_{2}$.

\subsection{Equivalence with reduced forms}

In this subsection, we will define the concept of a reduced form of a financial structure. Then we will give a result on the equivalence of a financial structure with its reduced forms. An important motivation for studying the reduced forms of a financial structure is the following.

To get existence of a financial equilibrium, we use a fixed point argument, so we need the compactness of attainable portfolios. With a financial structure, we may have unbounded attainable portfolios due to redundant assets ${ }^{4}$. So, a way to get an equilibrium is:

- considering a reduced form by removing redundant assets;

- then obtaining bounded attainable portfolios for the reduced form;

- then proving the existence for the reduced form;

- by equivalence, getting an equilibrium for the original economy.

Definition 4.2. Let $p \in \mathbb{R}^{\mathbb{L}}$ be a spot price vector. Let $\mathcal{F}=\left(\mathcal{J},(\xi(j))_{j \in \mathcal{J}}, V\right)$ be a financial structure. We call a reduced form of $\mathcal{F}$ with respect to $p$, any financial sub-structure $\mathcal{F}^{\prime}=\left(\mathcal{J}^{\prime},(\xi(j))_{j \in \mathcal{J}^{\prime}}, V^{\prime}\right)$ of $\mathcal{F}$ such that $\operatorname{rank} V(p)=$ $\operatorname{rank} V^{\prime}(p)=\# \mathcal{J}^{\prime}$.

If for all $p \in \mathbb{R}^{\mathbb{L}}$ we have $\operatorname{rank} V(p)=\operatorname{rank} V^{\prime}(p)=\# \mathcal{J}^{\prime}$, we say then that $\mathcal{F}^{\prime}$ is a reduced form of $\mathcal{F}$.

We note that according to Definition 4.2, we obtain a reduced form of a financial structure with respect to a spot price $p$ by simply eliminating the maximum number of redundant assets for $p$.

Remark 4.4. This example provides a financial structure which is not equivalent to a reduced form. Indeed, let $\mathcal{F}$ be a financial structures with $\mathbb{D}=$ $\left\{\xi_{0}, \xi_{1}, \xi_{2}, \xi_{3}\right\}$ as in Remark 4.1, $\mathcal{J}=\left\{j^{1}, j^{2}, j^{3}\right\}, \xi\left(j^{1}\right)=\xi_{0}, \xi\left(j^{2}\right)=\xi_{1}$ and $\xi\left(j^{3}\right)=\xi_{2}$. The payoff matrix is

$$
V=\left(\begin{array}{lll}
0 & 0 & 0 \\
1 & 0 & 0 \\
1 & 0 & 0 \\
1 & 1 & 1
\end{array}\right) \begin{aligned}
& \xi_{0} \\
& \xi_{1} \\
& \xi_{3}
\end{aligned}
$$

The matrix of a reduced form $\mathcal{F}^{\prime}$ of $\mathcal{F}$ is $V^{\prime}=\left(\begin{array}{ll}0 & 0 \\ 1 & 0 \\ 1 & 0 \\ 1 & 1\end{array}\right)$.

\footnotetext{
${ }^{4}$ Recall that an asset $j_{0}$ of a financial structure $\mathcal{F}=\left(\mathcal{J},(\xi(j))_{j \in \mathcal{J}}, V\right)$ is redundant at the spot price $p$ if the column vector $V^{j_{0}}(p)$ representing its payoffs on $\mathbb{D}$ is a linear combination of the other column vectors of the matrix $V$.

${ }^{5}$ See Definition 3.2.
} 
Let $\lambda=\left(\lambda_{0}, \lambda_{1}, \lambda_{2}, \lambda_{3}\right) \in \mathbb{R}_{++}^{4}$ and $\left(q, q^{\prime}\right) \in \mathbb{R}^{3} \times \mathbb{R}^{2}$ the associated arbitrage free prices. We have

$$
W(q)=\left(\begin{array}{ccc|l}
-\frac{\lambda_{1}+\lambda_{2}+\lambda_{3}}{\lambda_{0}} & 0 & 0 \\
1 & -\frac{\lambda_{3}}{\lambda_{1}} & 0 & \xi_{0} \\
1 & 0 & -\frac{\lambda_{3}}{\lambda_{2}} & \xi_{1} \\
\xi_{2} \\
1 & 1 & 1
\end{array}\right) \begin{aligned}
& \xi_{3} \\
& 1
\end{aligned}
$$

while the rank of the full payoff matrix $W^{\prime}\left(q^{\prime}\right)$ is at most equal to two, so $\operatorname{Im} W(q) \neq \operatorname{Im} W^{\prime}\left(q^{\prime}\right)$ hence $\mathcal{F}$ is not equivalent to $\mathcal{F}^{\prime}$.

The next proposition shows that Assumption $\mathbf{R}$ borrowed from [7] and stated in Subsection 4.2 is a sufficient condition to get the equivalence between a financial structure and its financial sub-structures when the ranges of the payoff matrices are equal. The proof is given later.

Proposition 4.4. Let $p \in \mathbb{R}^{\mathbb{L}}$ be a spot price vector. Let $\mathcal{F}=\left(\mathcal{J},(\xi(j))_{j \in \mathcal{J}}, V\right)$ be a financial structure satisfying Assumption $\boldsymbol{R}$ at the spot price $p$ and let $\mathcal{F}^{\prime}=$ $\left(\mathcal{J}^{\prime},(\xi(j))_{j \in \mathcal{J}^{\prime}}, V^{\prime}\right)$ be a financial sub-structure of $\mathcal{F}$. The following assertions are equivalent:

1) $\mathcal{F} \simeq_{p} \mathcal{F}^{\prime}$

2) For every state price $\lambda \in \mathbb{R}_{++}^{\mathbb{D}}, \operatorname{Im} W(p, q)=\operatorname{Im} W^{\prime}\left(p, q^{\prime}\right)$ where $q$ and $q^{\prime}$ are the associated arbitrage free prices;

3) For every state price $\lambda \in \mathbb{R}_{++}^{\mathbb{D}}$, $\operatorname{rank} W(p, q)=\operatorname{rank} W^{\prime}\left(p, q^{\prime}\right)$ where $q$ and $q^{\prime}$ are the associated arbitrage free prices;

4) $\operatorname{rank} V(p)=\operatorname{rank} V^{\prime}(p)$;

5) $\operatorname{Im} V(p)=\operatorname{Im} V^{\prime}(p)$;

6) $\forall \xi \in \mathbb{D}^{e}, \operatorname{Im} V^{\mathcal{J}(\xi)}(p)=\operatorname{Im} V^{\prime \mathcal{J}^{\prime}(\xi)}(p)$.

Note that (6) means that $\mathcal{F}$ and $\mathcal{F}^{\prime}$ satisfy Assumption $\mathbf{R} \mathbf{1}$ at $p$. So, under Assumption $\mathbf{R}$, a sub-structure is equivalent if we remove only redundant assets.

The following corollary is a direct consequence of the previous proposition because we consider a reduced form which is a particular case of a sub-structure and the rank of the payoff matrices are the same by definition.

Corollary 4.2. Given a spot price vector $p \in \mathbb{R}^{\mathbb{L}}$, let $\mathcal{F}=\left(\mathcal{J},(\xi(j))_{j \in \mathcal{J}}, V\right)$ be a financial structure satisfying Assumption $\boldsymbol{R}$ at the spot price $p$. Then, $\mathcal{F}$ is equivalent to each of its reduced forms.

Since Assumption $\mathbf{R}$ is always true in the case of a financial structure consisting only of short-term assets, we deduce from Corollary 4.2 the following corollary:

Corollary 4.3. A financial structure consisting only of short-term assets is equivalent to each of its reduced forms. 
Corollary 4.3 generalizes Proposition 1.5 of the thesis Zaier Aouani [2], which deals with the two-period case. The following corollary is deduced from Corollary 4.2 and Example 3.4.

Corollary 4.4. Given a spot price vector $p \in \mathbb{R}^{\mathbb{L}}$, let $\mathcal{F}_{1}=\left(\mathcal{J}_{1},(\xi(j))_{j \in \mathcal{J}_{1}}, V^{1}\right)$ and $\mathcal{F}_{2}=\left(\mathcal{J}_{2},(\xi(j))_{j \in \mathcal{J}_{2}}, V^{2}\right)$ be two financial structure satisfying Assumption $\boldsymbol{R}$ at the spot price $p$. Then for all reduced forms $\mathcal{F}_{1}^{\prime}$ of $\mathcal{F}_{1}$ with respect to $p$ and for all reduced forms $\mathcal{F}_{2}^{\prime}$ of $\mathcal{F}_{2}$ with respect to $p$ we have

$$
\mathcal{F}_{1} \cup \mathcal{F}_{2} \simeq_{p} \mathcal{F}_{1}^{\prime} \cup \mathcal{F}_{2}^{\prime}
$$

Remark 4.5. The following example shows that Assumption $\mathbf{R}$ is not necessary to get the equivalence between a financial structure and its reduced forms.

Indeed, let $\mathcal{F}=\left(\mathcal{J},(\xi(j))_{j \in \mathcal{J}}, V\right)$ be a financial structures such that $\mathbb{D}=$ $\left\{\xi_{0}, \xi_{1}, \xi_{2}, \xi_{3}\right\}$ as in Remark 4.1, $\mathcal{J}=\left\{j^{1}, j^{2}, j^{3}, j^{4}\right\}$ and $\xi\left(j^{1}\right)=\xi\left(j^{2}\right)=\xi\left(j^{3}\right)=$ $\xi_{0}, \xi\left(j^{4}\right)=\xi_{2}$. The payoff matrix is

$$
V=\left(\begin{array}{llll}
0 & 0 & 0 & 0 \\
1 & 0 & 0 & 0 \\
0 & 1 & 1 & 0 \\
0 & 1 & 0 & 1
\end{array}\right) \xi_{0}
$$

Assumption $\mathbf{R}$ is not satisfied since Vect $V_{\mathbb{D}^{+}\left(\xi_{2}\right)}^{\mathcal{J}\left(\mathbb{D}^{-}\left(\xi_{2}\right)\right)} \cap$ Vect $V_{\mathbb{D}^{+}\left(\xi_{2}\right)}^{\mathcal{J}\left(\xi_{2}\right)}=\mathbb{R} \neq\{0\}$. The financial structure $\mathcal{F}$ has exactly three reduced forms denoted by $\mathcal{F}^{1}, \mathcal{F}^{2}$ and $\mathcal{F}^{3}$.

$$
V^{1}=\left(\begin{array}{lll}
0 & 0 & 0 \\
1 & 0 & 0 \\
0 & 1 & 0 \\
0 & 1 & 1
\end{array}\right), V^{2}=\left(\begin{array}{lll}
0 & 0 & 0 \\
1 & 0 & 0 \\
0 & 1 & 1 \\
0 & 0 & 1
\end{array}\right) \text { and } V^{3}=\left(\begin{array}{ccc}
0 & 0 & 0 \\
1 & 0 & 0 \\
0 & 1 & 0 \\
0 & 0 & 1
\end{array}\right)
$$

The full payoff matrices are as follows: $W()=.\left(\begin{array}{cccc}\cdot & . & . & 0 \\ 1 & 0 & 0 & 0 \\ 0 & 1 & 1 & . \\ 0 & 1 & 0 & 1\end{array}\right)$,

$$
W^{1}(.)=\left(\begin{array}{ccc}
. & . & 0 \\
1 & 0 & 0 \\
0 & 1 & . \\
0 & 1 & 1
\end{array}\right), W^{2}(.)=\left(\begin{array}{ccc}
. & . & . \\
1 & 0 & 0 \\
0 & 1 & 1 \\
0 & 0 & 1
\end{array}\right) \text { and } W^{3}(.)=\left(\begin{array}{ccc}
. & . & 0 \\
1 & 0 & 0 \\
0 & 1 & . \\
0 & 0 & 1
\end{array}\right)
$$

Let $\lambda=\left(\lambda_{0}, \lambda_{1}, \lambda_{2}, \lambda_{3}\right) \in \mathbb{R}_{++}^{4}$ and $\left(q, q^{1}\right) \in \mathbb{R}^{4} \times \mathbb{R}^{3}$ be the associated arbitrage free prices. Then, $\operatorname{Im} W(q)=\operatorname{Im} W^{1}\left(q^{1}\right)$. Indeed, the column vectors of the matrix $W^{1}\left(q^{1}\right)$ are also column vectors of the matrix $W(q)$ so, Vect $W^{1}\left(q^{1}\right) \subset$ Vect $W(q)$. Moreover, $\operatorname{rank} W^{1}\left(q^{1}\right)=\operatorname{rank} W(q)=3$. Hence $\mathcal{F}^{1}$ is equivalent to $\mathcal{F}$. Similarly, we can show that the structures $\mathcal{F}^{2}$ and $\mathcal{F}^{3}$ are equivalent to $\mathcal{F}$.

Proof of Proposition 4.4. 1) implies 2). By definition of the equivalence of two financial structures. 2) implies 3). Because $\operatorname{rank} W(p, q)=\operatorname{dim} \operatorname{Im} W(p, q)$ and $\left.\operatorname{rank} W^{\prime}\left(p, q^{\prime}\right)=\operatorname{dim} \operatorname{Im} W^{\prime}\left(p, q^{\prime}\right) .3\right)$ implies 4$)$. Thanks to Assumption $\mathbf{R}($ See Proposition 3.5 and Lemma 3.1 in [7]). 4) implies 5). Because by definition 
of a sub-structure of a financial structure, we have always $\operatorname{Im} V^{\prime}(p) \subset \operatorname{Im} V(p)$. $5)$ implies 6). By definition of a sub-structure of a financial structure, we have always for all $\xi \in \mathbb{D}^{e}, \operatorname{Im} V^{\prime \mathcal{J}^{\prime}(\xi)}(p) \subset \operatorname{Im} V^{\mathcal{J}(\xi)}(p)$, hence $\operatorname{rank} V^{\prime \mathcal{J}^{\prime}(\xi)}(p) \leq$ $\operatorname{rank} V^{\mathcal{J}(\xi)}(p)$. Furthermore, in the proof of Proposition 3.5 in [7], it is shown that Assumption $\mathbf{R}$ implies $\operatorname{rank} V(p)=\sum_{\xi \in \mathbb{D} e} \operatorname{rank} V^{\mathcal{J}(\xi)}(p) . \mathcal{F}^{\prime}$ satisfies Assumption $\mathbf{R}$ as sub-structure of a financial structure satisfying assumption $\mathbf{R}$, so, we also have $\operatorname{rank} V^{\prime}(p)=\sum_{\xi \in \mathbb{D}^{e}} \operatorname{rank} V^{\prime \mathcal{J}^{\prime}(\xi)}(p)$. Hence $\sum_{\xi \in \mathbb{D}^{e}} \operatorname{rank} V^{\mathcal{J}(\xi)}(p)=$ $\sum_{\xi \in \mathbb{D}^{e}} \operatorname{rank} V^{\prime \mathcal{J}^{\prime}(\xi)}(p)$. Combining with the above inequalities, we conclude that $\operatorname{rank} V^{\mathcal{J}^{\prime}(\xi)}(p)=\operatorname{rank} V^{\mathcal{J}(\xi)}(p)$ for all $\xi \in \mathbb{D}^{e}$, and finally $\operatorname{Im} V^{\prime \mathcal{J}^{\prime}(\xi)}(p)=$ $\left.\operatorname{Im} V^{\mathcal{J}(\xi)}(p) ; 6\right)$ implies 1): thanks to Proposition 4.1.

\subsection{Equivalence for complete financial structures}

In Remark 4.2, the example shows that even if Assumption $\mathbf{R}$ is satisfied by both financial structures, $\operatorname{Im} V^{1}=\operatorname{Im} V^{2}$ and the sets of issuance nodes are equal i.e., $\mathbb{D}_{1}^{e}=\mathbb{D}_{2}^{e}$, the financial structures may not be equivalent. Note that the two financial structures satisfy also the stronger condition of Cornet and Ranjan [8], namely

$$
\forall \xi \in \mathbb{D} \text { and } \forall j, \ell \in \mathcal{J}, V_{\xi}^{j} V_{\xi}^{\ell}=0 \text { if } \xi(j) \neq \xi(\ell)
$$

Nevertheless, we can obtain a more accurate result for complete financial structures. Indeed, Assumption $\mathbf{R}$ is then sufficient to get the equivalence. Completeness means that all financial transfers are possible through the financial structure.

Definition 4.3. Let $\mathcal{F}=\left(\mathcal{J},(\xi(j))_{j \in \mathcal{J}}, V\right)$ be a financial structure. Given a spot price $p \in \mathbb{R}^{\mathbb{L}}$ and an arbitrage free price $q$, the financial structure $\mathcal{F}$ is complete at $(p, q)$ if $\operatorname{dim} \operatorname{Im} W(p, q)=\# \mathbb{D}-1$. If $\operatorname{dim} \operatorname{Im} W(p, q)<\# \mathbb{D}-1$ the financial structure $\mathcal{F}$ is incomplete at $(p, q)$.

The following proposition, which is a direct consequence of Proposition 3.5 in [7], gives a characterization of market completeness under Assumption $\mathbf{R}$.

Proposition 4.5. (Complete Markets) Given a spot price $p \in \mathbb{R}^{\mathbb{L}}$, let $\mathcal{F}=$ $\left(\mathcal{J},(\xi(j))_{j \in \mathcal{J}}, V\right)$ be a financial structure satisfying Assumption $\boldsymbol{R}$ at the spot price p. If $\operatorname{rank} V(p)=\# \mathbb{D}-1$ then, for all state price $\lambda \in \mathbb{R}_{++}^{\mathbb{D}}$, the financial structure $\mathcal{F}$ is complete at $(p, q)$, where $q$ is the arbitrage free asset price associated to $\lambda$.

The following proposition gives a result of equivalence among complete financial structures under Assumption R.

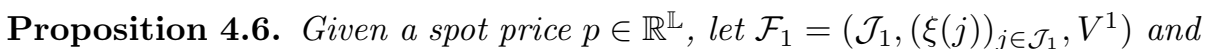

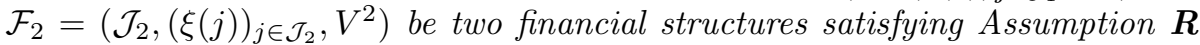
at the spot price $p$. Let us assume that $\operatorname{rank} V^{1}(p)=\# \mathbb{D}-1$. The following assertions are equivalent:

(a) $\mathcal{F}_{1} \simeq p \mathcal{F}_{2}$; 
(b) For all state price $\lambda \in \mathbb{R}_{++}^{\mathbb{D}}$, the financial structure $\mathcal{F}_{1}$ /resp. $\mathcal{F}_{2} /$ is complete at $\left(p, q^{1}\right)$ [resp. $\left.\left(p, q^{2}\right)\right]$ where $\left(q_{1}, q_{2}\right)$ is the couple of arbitrage free prices associated to $\lambda$;

(c) $\operatorname{rank} V^{1}(p)=\operatorname{rank} V^{2}(p)$;

(d) $\operatorname{Im} V^{1}(p)=\operatorname{Im} V^{2}(p)$.

Proof of Proposition 4.6. (a) implies (b). $\mathcal{F}_{1} \simeq_{p} \mathcal{F}_{2}$ implies for all state price $\lambda \in \mathbb{R}_{++}^{\mathbb{D}}, \operatorname{Im} W^{1}\left(p, q^{1}\right)=\operatorname{Im} W^{2}\left(p, q^{2}\right)$. Since Assumption $\mathbf{R}$ holds true for both financial structure, thanks to Proposition 3.5 in [7], $\# \mathbb{D}-1=\operatorname{rank} V^{1}(p)=$ $\operatorname{rank} W^{1}\left(p, q^{1}\right)=\operatorname{dim} \operatorname{Im} W^{1}\left(p, q^{1}\right)=\operatorname{dim} \operatorname{Im} W^{2}\left(p, q^{2}\right)=\operatorname{rank} V^{2}(p)$. So, the financial structure $\mathcal{F}_{1}$ [resp. $\mathcal{F}_{2}$ ] is complete at $\left(p, q^{1}\right)$ [resp. $\left.\left(p, q^{2}\right)\right]$.

(b) implies (c) thanks to Proposition 3.5 in [7].

Let us now show that (c) implies (d). By assumption, $\operatorname{rank} V^{1}(p)=\# \mathbb{D}-1$ and, since the first row of $V^{1}(p)$ has only zero entries, $\operatorname{Im} V^{1}(p)=\left\{y \in \mathbb{R}^{\mathbb{D}} \mid\right.$ $\left.y_{\xi_{0}}=0\right\}$. The same reasoning applies to $V^{2}(p)$ so $\operatorname{Im} V^{1}(p)=\operatorname{Im} V^{2}(p)$.

Let us show now that $(d)$ implies $(a)$. Let $\lambda \in \mathbb{R}_{++}^{\mathbb{D}}$ be a state price. Let $\hat{q}^{1} \in \mathbb{R}^{\mathcal{J}_{1}}$ and $\hat{q}^{2} \in \mathbb{R}^{\mathcal{J}_{2}}$ the two arbitrage free prices associated with $\lambda$. Since Assumption $\mathbf{R}$ holds true for both financial structure, thanks to Proposition 3.5 in [7], $(d)$ implies that $\# \mathbb{D}-1=\operatorname{rank} V^{1}(p)=\operatorname{rank} W^{1}\left(p, \hat{q}^{1}\right)=\operatorname{rank} V^{2}(p)=$ $\operatorname{rank} W^{2}\left(p, \hat{q}^{2}\right)$. So since $\operatorname{Im} W^{1}\left(p, \hat{q}^{1}\right)$ and $\operatorname{Im} W^{2}\left(p, \hat{q}^{2}\right)$ are included in $\lambda^{\perp}$, one concludes that $\operatorname{Im} W^{1}\left(p, \hat{q}^{1}\right)=\operatorname{Im} W^{2}\left(p, \hat{q}^{2}\right)=\lambda^{\perp}$. Consequently, $\mathcal{F}_{1} \simeq_{p} \mathcal{F}_{2}$.

Remark 4.6. In the two-period case, if there exists an arbitrage free price for which the financial market is complete, the financial structure is complete for any other arbitrage free price. With several periods, if the assets are all shortterm, this result is deduced of Proposition 5.2 of Angeloni and Cornet in [1]. But if there are long-term assets in the financial structure, Proposition 4.6 is no more true without Assumption R. Indeed, consider a stochastic economy with $T=2$ and tree nodes, namely $\mathbb{D}=\left\{\xi_{0}, \xi_{1}, \xi_{2}\right\}$ and let $\mathcal{F}_{1}=\left(\mathcal{J}_{1},(\xi(j))_{\left.j \in \mathcal{J}_{1}, V^{1}\right)}\right.$ and $\mathcal{F}_{2}=\left(\mathcal{J}_{2},(\xi(j))_{j \in \mathcal{J}_{2}}, V^{2}\right)$ be two financial structures. In the first structure there are two assets $j_{1}^{1}$ and $j_{1}^{2}$ with $\xi\left(j_{1}^{1}\right)=\xi_{0}$ and $\xi\left(j_{1}^{2}\right)=\xi_{1}$. In the second structure there are two assets $j_{2}^{1}$ and $j_{2}^{2}$ with $\xi\left(j_{2}^{1}\right)=\xi\left(j_{2}^{2}\right)=\xi_{0}$.

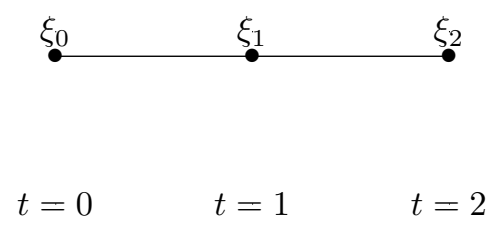

Figure 5: the tree $\mathbb{D}$

Then the payoff matrices are:

$$
V^{1}=\left(\begin{array}{cc}
0 & 0 \\
-1 & 0 \\
1 & 1
\end{array}\right) \begin{aligned}
& \xi_{0} \\
& \xi_{1} \\
& \xi_{2}
\end{aligned} \quad \text { and } \quad V^{2}=\left(\begin{array}{cc}
0 & 0 \\
1 & 0 \\
0 & 1
\end{array}\right) \begin{aligned}
& \xi_{0} \\
& \xi_{1} \\
& \xi_{2}
\end{aligned}
$$


Assumption $\mathbf{R}$ is not satisfied by $\mathcal{F}_{1}$. It is clear that

$$
\operatorname{rank} V^{1}(p)=\operatorname{rank} V^{2}(p)=\# \mathbb{D}-1=2 .
$$

Let $\lambda=(1,1,1), \hat{q}^{1}=(0,1)$ [resp. $\hat{q}^{2}=(1,1)$ ] is the arbitrage free price of $\mathcal{F}_{1}$ [resp. $\mathcal{F}_{2}$ ] associated with $\lambda$. The full payoff matrices associated with $\hat{q}^{1}$ and $\hat{q}^{2}$ are:

$$
W^{1}\left(\hat{q}^{1}\right)=\left(\begin{array}{cc}
\mathbf{0} & 0 \\
-1 & \mathbf{- 1} \\
1 & 1
\end{array}\right) \begin{aligned}
& \xi_{0} \\
& \xi_{1} \\
& \xi_{2}
\end{aligned} \text { and } W^{2}\left(\hat{q}^{2}\right)=\left(\begin{array}{cc}
\mathbf{- 1} & \mathbf{- 1} \\
1 & 0 \\
0 & 1
\end{array}\right) \begin{aligned}
& \xi_{0} \\
& \xi_{1} \\
& \xi_{2}
\end{aligned}
$$

We have $\operatorname{rank} W^{1}\left(\hat{q}^{1}\right)=1$ and $\operatorname{rank} W^{2}\left(\hat{q}^{2}\right)=2$ this implies that $\operatorname{Im} W^{1}\left(\hat{q}^{1}\right) \neq$ $\operatorname{Im} W^{2}\left(\hat{q}^{2}\right)$. So the structures $\mathcal{F}_{1}$ and $\mathcal{F}_{2}$ are not equivalent and the financial structure $\mathcal{F}_{1}$ is not complete at $\hat{q}^{1}$.

Remark 4.7. Without the completeness, even if Assumption $\mathbf{R}$ is satisfied in the two financial structures and there is a state price $\lambda \in \mathbb{R}_{++}^{\mathbb{D}}$ such that $\operatorname{Im} W^{1}\left(p, q^{1}\right)=\operatorname{Im} W^{2}\left(p, q^{2}\right)$ for the associated arbitrage free asset prices, we have no guarantee that the two structures are equivalent at $p$. Indeed, let $\mathbb{D}=$ $\left\{\xi_{0}, \xi_{1}, \xi_{2}, \xi_{3}, \xi_{4}\right\}, \mathcal{J}_{1}=\left\{j_{1}^{1}, j_{1}^{2}, j_{1}^{3}\right\}, \mathcal{J}_{2}=\left\{j_{2}^{1}, j_{2}^{2}, j_{2}^{3}\right\} \cdot \xi\left(j_{1}^{1}\right)=\xi\left(j_{1}^{2}\right)=\xi\left(j_{2}^{1}\right)=$ $\xi_{0}$ and $\xi\left(j_{1}^{3}\right)=\xi\left(j_{2}^{2}\right)=\xi\left(j_{2}^{3}\right)=\xi_{1}$.

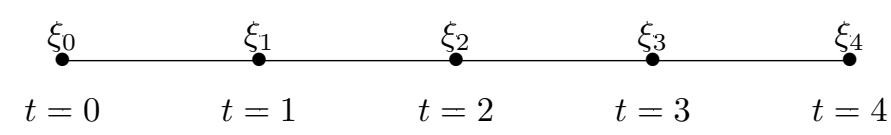

Figure 6: the tree $\mathbb{D}$

The payoff matrices and the full payoff matrices for the state price $\lambda=$ $(1,1,1,1,1)$ are:

$$
\begin{aligned}
V^{1} & =\left(\begin{array}{ccc}
0 & 0 & 0 \\
1 & -1 & 0 \\
-1 & 1 & 1 \\
0 & -1 & 1 \\
0 & 1 & -2
\end{array}\right) \begin{array}{l}
\xi_{0} \\
\xi_{1} \\
\xi_{2} \\
\xi_{3} \\
\xi_{4}
\end{array} \text { and } W^{1}\left(q^{1}\right)=\left(\begin{array}{ccc}
\mathbf{0} & \mathbf{0} & 0 \\
1 & -1 & \mathbf{0} \\
-1 & 1 & 1 \\
0 & -1 & 1 \\
0 & 1 & -2
\end{array}\right) \begin{array}{l}
\xi_{0} \\
\xi_{1} \\
\xi_{2} \\
\xi_{3} \\
\xi_{4}
\end{array} \\
V^{2} & =\left(\begin{array}{ccc}
0 & 0 & 0 \\
1 & 0 & 0 \\
-1 & 1 & 1 \\
0 & -1 & 1 \\
0 & 1 & -2
\end{array}\right) \begin{array}{l}
\xi_{0} \\
\xi_{1} \\
\xi_{2} \\
\xi_{3} \\
\xi_{4}
\end{array} \text { and } W^{2}\left(q^{2}\right)=\left(\begin{array}{ccc}
\mathbf{0} & 0 & 0 \\
1 & -\mathbf{1} & \mathbf{0} \\
-1 & 1 & 1 \\
0 & -1 & 1 \\
0 & 1 & -2
\end{array}\right) \begin{array}{l}
\xi_{0} \\
\xi_{1} \\
\xi_{2} \\
\xi_{3} \\
\xi_{4}
\end{array}
\end{aligned}
$$

Assumption $\mathbf{R}$ is satisfied by the two financial structures and it is clear that $\operatorname{Im} W^{1}\left(q^{1}\right)=\operatorname{Im} W^{2}\left(q^{2}\right)$ since $W^{1}\left(q^{1}\right)=W^{2}\left(q^{2}\right)$.

Now let $\hat{\lambda}=(1,1,1,1,2)$ be another state price. Then, 


$$
W^{1}\left(\hat{q}^{1}\right)=\left(\begin{array}{ccc}
\mathbf{0} & -\mathbf{1} & 0 \\
1 & -1 & \mathbf{2} \\
-1 & 1 & 1 \\
0 & -1 & 1 \\
0 & 1 & -2
\end{array}\right) \begin{aligned}
& \xi_{0} \\
& \xi_{1} \\
& \xi_{2} \\
& \xi_{3} \\
& \xi_{4}
\end{aligned} \text { and } W^{1}\left(\hat{q}^{2}\right)=\left(\begin{array}{ccc}
\mathbf{0} & 0 & 0 \\
1 & -\mathbf{2} & \mathbf{2} \\
-1 & 1 & 1 \\
0 & -1 & 1 \\
0 & 1 & -2
\end{array}\right) \begin{aligned}
& \xi_{0} \\
& \xi_{1} \\
& \xi_{2} \\
& \xi_{3} \\
& \xi_{4}
\end{aligned}
$$

It is clear that $\operatorname{Im} W^{1}\left(\hat{q}^{1}\right) \neq \operatorname{Im} W^{2}\left(\hat{q}^{2}\right)$ since $W_{\xi_{0}}^{1}\left(\hat{q}^{1}\right) \neq 0$ and $W_{\xi_{0}}^{2}\left(\hat{q}^{2}\right)=0$. Consequently the financial structures $\mathcal{F}_{1}$ and $\mathcal{F}_{2}$ are not equivalent at $p$.

\section{Appendix}

\section{Proof of Examples of Subsection 3.1}

Example 3.1. Let $p$ be a spot price, $\lambda \in \mathbb{R}_{++}^{\mathbb{D}}$ be a state price, $\alpha$ be one non-zero real number and $\left(q, q^{\alpha}\right) \mathbb{R}^{\mathcal{J}} \times \mathbb{R}^{\mathcal{J}}$ be the couple of arbitrage free prices associated to $\lambda$. From the arbitrage free condition, $q^{\alpha}=\alpha q$, so $W^{\alpha}\left(p, q^{\alpha}\right)=\alpha W(p, q)$, which implies that $\operatorname{Im} W^{\alpha}\left(p, q^{\alpha}\right)=\operatorname{Im} W(p, q)$ since $\alpha \neq 0$. So, $\mathcal{F} \simeq \mathcal{F}_{\alpha}$.

Example 3.2. Let $p$ be a spot price, $\lambda \in \mathbb{R}_{++}^{\mathbb{D}}$ be a state price and $\left(q^{1}, q^{2}\right) \in$ $\mathbb{R}^{\mathcal{J}_{1}} \times \mathbb{R}^{\mathcal{J}_{2}}$ be the couple of arbitrage free prices associated to $\lambda$. From the structure of $V$, the arbitrage free price $q$ associated to $\lambda$ for the payoff matrix $V$ is $\left(q^{1}, q^{2}\right)$. So $W(p, q)=\left[W^{1}\left(p, q^{1}\right), W^{2}\left(p, q^{2}\right)\right]$. Consequently,

$$
\begin{aligned}
\operatorname{Im} W(p, q) & =\operatorname{Im} W^{1}\left(p, q^{1}\right)+\operatorname{Im} W^{2}\left(p, q^{2}\right) \\
& =\operatorname{Im} W^{2}\left(p, q^{2}\right)+\operatorname{Im} W^{1}\left(p, q^{1}\right) \\
& =\operatorname{Im}\left[W^{2}\left(p, q^{2}\right), W^{1}\left(p, q^{1}\right)\right]
\end{aligned}
$$

Hence, $\mathcal{F}_{1} \cup \mathcal{F}_{1} \simeq \mathcal{F}_{2} \cup \mathcal{F}_{1}$. Furthermore, if $\mathcal{F}_{1} \simeq \mathcal{F}_{2}$, then $\operatorname{Im} W^{1}\left(p, q^{1}\right)=$ $\operatorname{Im} W^{2}\left(p, q^{2}\right)$ and $\operatorname{Im} W^{1}\left(p, q^{1}\right)+\operatorname{Im} W^{2}\left(p, q^{2}\right)=\operatorname{Im} W^{1}\left(p, q^{1}\right)=\operatorname{Im} W^{2}\left(p, q^{2}\right)$. So, $\mathcal{F}_{1} \cup \mathcal{F}_{1} \simeq \mathcal{F}_{1} \simeq \mathcal{F}_{2}$.

Example 3.4. Let $\lambda \in \mathbb{R}_{++}^{\mathbb{D}}$ be a state price and $\left(q^{1}, q^{2}, q^{3}, q^{4}\right) \mathbb{R}^{\mathcal{J}_{1}} \times \mathbb{R}^{\mathcal{J}_{2}} \times$ $\mathbb{R}^{\mathcal{J}_{3}} \times \mathbb{R}^{\mathcal{J}_{4}}$ be the arbitrage free prices associated to $\lambda$. Like in the previous example, we remark that the full payoff matrix associated to $\mathcal{F}_{1} \cup \mathcal{F}_{3}$ (resp. $\left.\mathcal{F}_{2} \cup \mathcal{F}_{4}\right)$ is $\left[W^{1}\left(p, q^{1}\right), W^{3}\left(p, q^{3}\right)\right]$ (resp. $\left.\left[W^{2}\left(p, q^{1}\right), W^{4}\left(p, q^{3}\right)\right]\right)$. Furthermore,

$$
\begin{aligned}
& \operatorname{Im}\left[W^{1}\left(p, q^{1}\right), W^{3}\left(p, q^{3}\right)\right]=\operatorname{Im} W^{1}\left(p, q^{1}\right)+\operatorname{Im} W^{3}\left(p, q^{3}\right) \text { and } \\
& \operatorname{Im}\left[W^{2}\left(p, q^{2}\right), W^{4}\left(p, q^{4}\right)\right]=\operatorname{Im} W^{2}\left(p, q^{2}\right)+\operatorname{Im} W^{4}\left(p, q^{4}\right) .
\end{aligned}
$$

Since $\mathcal{F}_{1} \simeq_{p} \mathcal{F}_{2}$ and $\mathcal{F}_{3} \simeq_{p} \mathcal{F}_{4}$,

$$
\operatorname{Im} W^{1}\left(p, q^{1}\right)=\operatorname{Im} W^{2}\left(p, q^{2}\right) \text { and } \operatorname{Im} W^{3}\left(p, q^{3}\right)=\operatorname{Im} W^{4}\left(p, q^{4}\right) .
$$

Hence $\operatorname{Im}\left[W^{1}\left(p, q^{1}\right), W^{3}\left(p, q^{3}\right)\right]=\operatorname{Im}\left[W^{2}\left(p, q^{2}\right), W^{4}\left(p, q^{4}\right)\right]$, which shows that $\mathcal{F}_{1} \cup \mathcal{F}_{3} \simeq_{p} \mathcal{F}_{2} \cup \mathcal{F}_{4}$.

Proof of Proposition 3.3 Let $\mathcal{F}_{4}$ be the financial structure with the assets of $\mathcal{F}_{2}$, which are not in $\mathcal{F}_{3}$, that is, $\mathcal{F}_{4}=\left(\mathcal{J}_{4}=\mathcal{J}_{2} \backslash \mathcal{J}_{3},(\xi(j))_{j \in \mathcal{J}_{4}}, V^{4}=V^{\mathcal{J}_{4}}\right)$. So $\mathcal{F}_{2} \simeq \mathcal{F}_{3} \cup \mathcal{F}_{4}$. Then, for the spot price $p$, since $\mathcal{F}_{1} \simeq_{p} \mathcal{F}_{3}$, from Example 3.4 one gets that $\mathcal{F}_{1} \cup \mathcal{F}_{4} \simeq_{p} \mathcal{F}_{3} \cup \mathcal{F}_{4} \simeq_{p} \mathcal{F}_{2}$. 
Proof of Lemma 4.1. As already noticed, Assumption R1 implies the equality of the ranges of the payoff matrices. We now prove the converse.

First, we prove that the equality of the ranges of the matrices implies that the node of issuance are the same for both structures. Let us assume that there exists $\bar{\xi} \in \mathbb{D}_{1}^{e}$ and $\bar{\xi} \notin \mathbb{D}_{2}^{e}$. Let $j_{1} \in \mathcal{J}_{1}$ such that $\xi\left(j_{1}\right)=\bar{\xi}$. Since there is no trivial asset, there exists $\eta \in \xi^{+}$such that $V_{\eta}^{1 j_{1}}(p) \neq 0$. But, since $\bar{\xi} \notin \mathbb{D}_{2}^{e}$ and all assets are short term, for all $j \in \mathcal{J}_{2}, V_{\eta}^{2 j}(p)=0$. Consequently, $V^{1 j_{1}}(p)$ is not a linear combination of $\left(V^{2 j}(p)\right)_{j \in \mathcal{J}_{2}}$, which contradicts the fact that $\operatorname{Im} V^{1}(p)=\operatorname{Im} V^{2}(p)$. In the same way, one shows that there is no node in $\mathbb{D}_{2}^{e}$ which does not belong to $\mathbb{D}_{1}^{e}$. So, the issuance nodes are the same for both structures.

Now we show that the condition $\operatorname{Im} V^{1}(p)=\operatorname{Im} V^{2}(p)$ implies that Assumption $\mathbf{R} 1$ holds true at the spot price $p$. From [7], since there is only short-term assets, the two structures satisfies Assumption R, so

$$
\operatorname{Im} V^{1}(p)=\oplus_{\xi \in \mathbb{D}^{e}} \operatorname{Im} V^{1, \mathcal{J}_{1}(\xi)}(p)=\oplus_{\xi \in \mathbb{D}^{e}} \operatorname{Im} V^{2, \mathcal{J}_{2}(\xi)}(p)=\operatorname{Im} V^{2}(p)
$$

Let $\xi \in \mathbb{D}^{e}$ and $y \in \operatorname{Im} V^{1, \mathcal{J}_{1}(\xi)}(p)$. Since we have only short-term assets, $y_{\eta}=0$ for $\eta \notin \xi^{+}$. From the previous equality, there exists a unique couple $\left(z^{\xi}, z\right) \in$ $\operatorname{Im} V^{2, \mathcal{J}_{2}(\xi)}(p) \times \oplus_{\xi^{\prime} \in \mathbb{D}^{e}, \xi^{\prime} \neq \xi} \operatorname{Im} V^{2, \mathcal{J}_{2}\left(\xi^{\prime}\right)}(p)$ such that $y=z^{\xi}+z$. Once again, since we have only short-term assets, for all $\eta \in \xi^{+}, z_{\eta}=0$ since $\xi^{\prime} \neq \xi$. For all $\eta \notin \xi^{+}, y_{\eta}=0=z_{\eta}^{\xi}$ hence, $z_{\eta}=0$. So, $z=0$ and $y=z^{\xi} \in \operatorname{Im} V^{2, \mathcal{J}_{2}(\xi)}(p)$, which proves that $\operatorname{Im} V^{1, \mathcal{J}_{1}(\xi)}(p) \subset \operatorname{Im} V^{2, \mathcal{J}_{1}(\xi)}(p)$. The converse inclusion is proved in the same way, so Assumption R1 is satisfied.

Proof of Proposition 4.2 (i) This is obvious since all assets are issued at the same node.

(ii) From Proposition 3.5 in [7], Assumption $\mathbf{R}$ implies

$$
\operatorname{rank} V^{1}(p)=\sum_{\xi \in \mathbb{D}_{1}^{e}} \operatorname{rank} V^{1^{\mathcal{J}_{1}(\xi)}}(p) \text { and } \operatorname{rank} V^{2}(p)=\sum_{\xi \in \mathbb{D}_{2}^{e}} \operatorname{rank} V^{2^{\mathcal{J}_{2}(\xi)}}(p)
$$

Since by assumption $\operatorname{rank} V^{1}(p)=\operatorname{rank} V^{2}(p)$, we have:

$$
\begin{gathered}
\sum_{\xi \in \mathbb{D}_{1}^{e} \cup \mathbb{D}_{2}^{e}} \operatorname{dim} \operatorname{Im} V^{1^{\mathcal{J}_{1}(\xi)}}(p)=\sum_{\xi \in \mathbb{D}_{1}^{e} \cup \mathbb{D}_{2}^{e}} \operatorname{rank} V^{1^{\mathcal{J}_{1}}(\xi)}(p)= \\
\sum_{\xi \in \mathbb{D}_{1}^{e} \cup \mathbb{D}_{2}^{e}} \operatorname{rank} V^{2^{\mathcal{J}_{2}(\xi)}}(p)=\sum_{\xi \in \mathbb{D}_{1}^{e} \cup \mathbb{D}_{2}^{e}} \operatorname{dim} \operatorname{Im} V^{2} \mathcal{J}_{2}(\xi)(p)
\end{gathered}
$$

and by assumption we have for all $\xi \in \mathbb{D}_{1}^{e} \cup \mathbb{D}_{2}^{e}, \operatorname{Im} V^{1 \mathcal{J}_{1}(\xi)}(p) \subset \operatorname{Im} V^{2 \mathcal{J}_{2}(\xi)}(p)$ thus this implies that $\xi \in \mathbb{D}_{1}^{e} \cup \mathbb{D}_{2}^{e}, \operatorname{dim} \operatorname{Im} V^{1^{\mathcal{J}_{1}(\xi)}}(p) \leq \operatorname{dim} \operatorname{Im} V^{2 \mathcal{J}_{2}(\xi)}(p)$. Combined with the previous equality, this implies that $\operatorname{dim} \operatorname{Im} V^{\mathcal{J}_{1}(\xi)}(p)=$ $\operatorname{dim} \operatorname{Im} V^{2 \mathcal{J}_{2}(\xi)}(p)$, which together with $\operatorname{Im} V^{1^{\mathcal{J}_{1}(\xi)}}(p) \subset \operatorname{Im} V^{2^{\mathcal{J}_{2}(\xi)}}(p)$ leads to $\operatorname{Im} V^{1 \mathcal{J}_{1}(\xi)}(p)=\operatorname{Im} V^{2 \mathcal{J}_{2}(\xi)}(p)$. So Assumption $\mathbf{R} 1$ is true at the spot price $p$ 


\section{References}

[1] L. Angeloni and B. Cornet. Existence of financial equilibria in a multi-period stochastic economy. Mathematical Economics, 8:1-31, 2006.

[2] Z. Aouani. Financial Economies with Restricted Participation. PhD thesis, Faculty of the Graduate School of the University of Kansas, 2008.

[3] Z. Aouani and B. Cornet. Characterizing reduced financial structures. Working Paper, University of Paris 1, 2008.

[4] Z. Aouani and B. Cornet. Equivalent reduced form of a financial structure. Working Paper, University of Paris 1, 2008.

[5] Z. Aouani and B. Cornet. Existence of financial equilibria with restricted participations. Journal of Mathematical Economics, 45:772-786, 2009.

[6] J-M. Bonnisseau and A. Chéry. On the equivalence of financial structures with short-term assets. Working Paper, University of Paris 1, 2013.

[7] J-M. Bonnisseau and A. Chéry. Stability of marketable payoffs with longterm assets. Working Paper, University of Paris 1, 2013.

[8] B. Cornet and A. Ranjan. A remark on arbitrage free prices in multi-period economy. Working Paper, University of Paris 1, 2012.

[9] M. Magill and M. Quinzii. Theory of Incomplete Markets. Cambridge, 1996. 